\title{
Existence and concentration phenomena for a class of indefinite variational problems with critical growth
}

\author{
Claudianor O. Alves*, Geilson F. Germano ${ }^{\dagger}$ \\ Universidade Federal de Campina Grande \\ Unidade Acadêmica de Matemática \\ CEP: 58429-900, Campina Grande - Pb, Brazil
}

\begin{abstract}
In this paper we are interested to prove the existence and concentration of ground state solution for the following class of problems

$$
-\Delta u+V(x) u=A(\epsilon x) f(u), \quad x \in \mathbb{R}^{N}, \quad(P)_{\epsilon}
$$

where $N \geq 2, \epsilon>0, A: \mathbb{R}^{N} \rightarrow \mathbb{R}$ is a continuous function that satisfies

$$
0<\inf _{x \in \mathbb{R}^{N}} A(x) \leq \lim _{|x| \rightarrow+\infty} A(x)<\sup _{x \in \mathbb{R}^{N}} A(x)=A(0)
$$

$f: \mathbb{R} \rightarrow \mathbb{R}$ is a continuous function having critical growth, $V: \mathbb{R}^{N} \rightarrow \mathbb{R}$ is a continuous and $\mathbb{Z}^{N}$-periodic function with $0 \notin \sigma(\Delta+V)$. By using variational methods, we prove the existence of solution for $\epsilon$ small enough. After that, we show that the maximum points of the solutions concentrate around of a maximum point of $A$.
\end{abstract}

Mathematics Subject Classifications (2010): 35B40, 35J2, 47A10 .

Keywords: Concentration of solutions, Variational methods, Indefinite strongly functional, Critical growth.

\footnotetext{
${ }^{*}$ C. O. Alves was partially supported by CNPq/Brazil 301807/2013-2 and INCT-MAT, coalves@mat.ufcg.edu.br

${ }^{\dagger}$ G. F. Germano was partially supported by CAPES, geilsongermano@hotmail.com
} 


\section{Introduction}

This paper concerns with the existence and concentration of ground state solution for the semilinear Schrödinger equation

$$
\left\{\begin{array}{l}
-\Delta u+V(x) u=A(\epsilon x) f(u), \quad x \in \mathbb{R}^{N}, \\
u \in H^{1}\left(\mathbb{R}^{N}\right),
\end{array}\right.
$$

where $N \geq 2, \epsilon$ is a positive parameter, $f: \mathbb{R} \rightarrow \mathbb{R}$ is a continuous function with critical growth and $V, A: \mathbb{R} \rightarrow \mathbb{R}$ are continuous functions verifying some technical conditions.

In whole this paper, $V$ is $\mathbb{Z}^{N}$-periodic with

$$
0 \notin \sigma(-\Delta+V), \quad \text { the spectrum of }-\Delta+V,
$$

which becomes the problem strongly indefinite. Related to the function $A$, we assume that it is a continuous function satisfying

$$
0<A_{0}=\inf _{x \in \mathbb{R}^{N}} A(x) \leq \lim _{|x| \rightarrow+\infty} A(x)=A_{\infty}<\sup _{x \in \mathbb{R}^{N}} A(x) .
$$

The present article has as first motivation some recent articles that have studied the existence of ground state solution for related problems with $(P)_{\epsilon}$, more precisely for strongly indefinite problems of the type

$$
\left\{\begin{array}{l}
-\Delta u+V(x) u=f(x, u), \quad x \in \mathbb{R}^{N}, \\
u \in H^{1}\left(\mathbb{R}^{N}\right) .
\end{array}\right.
$$

In [13], Kryszewski and Szulkin have studied the existence of ground state solution for $\left(P_{1}\right)$ by supposing the condition $(V)$. Related to the function $f: \mathbb{R}^{N} \times \mathbb{R} \rightarrow \mathbb{R}$, they assumed that $f$ is continuous, $\mathbb{Z}^{N}$-periodic in $x$ with

$$
|f(x, t)| \leq c\left(|t|^{q-1}+|t|^{p-1}\right), \quad \forall t \in \mathbb{R} \quad \text { and } \quad x \in \mathbb{R}^{N}
$$

and

$$
0<\alpha F(x, t) \leq t f(x, t) \quad \forall(x, t) \in \mathbb{R}^{N} \times \mathbb{R}^{*}, \quad F(x, t)=\int_{0}^{t} f(x, s) d s \quad\left(h_{2}\right)
$$

for some $c>0, \alpha>2$ and $2<q<p<2^{*}$ where $2^{*}=\frac{2 N}{N-2}$ if $N \geq 3$ and $2^{*}=+\infty$ if $N=1,2$. The above hypotheses guarantee that the energy functional associated with $\left(P_{1}\right)$ given by

$$
J(u)=\frac{1}{2} \int_{\mathbb{R}^{N}}\left(|\nabla u|^{2}+V(x)|u|^{2}\right) d x-\int_{\mathbb{R}^{N}} F(x, u) d x, \forall u \in H^{1}\left(\mathbb{R}^{N}\right),
$$


is well defined and belongs to $C^{1}\left(H^{1}\left(\mathbb{R}^{N}\right), \mathbb{R}\right)$. By $(V)$, there is an equivalent inner product $\langle$,$\rangle in H^{1}\left(\mathbb{R}^{N}\right)$ such that

$$
J(u)=\frac{1}{2}\left\|u^{+}\right\|^{2}-\frac{1}{2}\left\|u^{-}\right\|^{2}-\int_{\mathbb{R}^{N}} F(x, u) d x,
$$

where $\|u\|=\sqrt{\langle u, u\rangle}$ and $H^{1}\left(\mathbb{R}^{N}\right)=E^{+} \oplus E^{-}$corresponds to the spectral decomposition of $-\Delta+V$ with respect to the positive and negative part of the spectrum with $u=u^{+}+u^{-}$, where $u^{+} \in E^{+}$and $u^{-} \in E^{-}$. In order to show the existence of solution for $\left(P_{1}\right)$, Kryszewski and Szulkin introduced a new and interesting generalized link theorem. In [15], Li and Szulkin have improved this generalized link theorem to prove the existence of solution for a class of strongly indefinite problem with $f$ being asymptotically linear at infinity.

The link theorems above mentioned have been used in a lot of papers, we would like to cite Chabrowski and Szulkin [5], do Ó and Ruf [8], Furtado and Marchi [9], Tang [23, 24] and their references.

Pankov and Pflüger [17] also have considered the existence of solution for problem $\left(P_{1}\right)$ with the same conditions considered in [13], however the approach is based on an approximation technique of periodic function together with the linking theorem due to Rabinowitz [20]. After, Pankov [16] has studied the existence of solution for problems of the type

$$
\left\{\begin{array}{l}
-\Delta u+V(x) u= \pm f(x, u), \quad x \in \mathbb{R}^{N} \\
u \in H^{1}\left(\mathbb{R}^{N}\right)
\end{array}\right.
$$

by supposing $(V),\left(h_{1}\right)-\left(h_{2}\right)$ and employing the same approach explored in [17. In [16] and [17, the existence of ground state solution has been established by supposing that $f$ is $C^{1}$ and there is $\theta \in(0,1)$ such that

$$
0<t^{-1} f(x, t) \leq \theta f_{t}^{\prime}(x, t), \quad \forall t \neq 0 \quad \text { and } \quad x \in \mathbb{R}^{N} .
$$

However, in [16], Pankov has found a ground state solution by minimizing the energy functional $J$ on the set

$$
\mathcal{O}=\left\{u \in H^{1}\left(\mathbb{R}^{N}\right) \backslash E^{-} ; J^{\prime}(u) u=0 \text { and } J^{\prime}(u) v=0, \forall v \in E^{-}\right\} .
$$

The reader is invited to see that if $J$ is strongly definite, that is, when $E^{-}=\{0\}$, the set $\mathcal{O}$ is exactly the Nehari manifold associated with $J$. Hereafter, we say that $u_{0} \in H^{1}\left(\mathbb{R}^{N}\right)$ is a ground state solution if

$$
J^{\prime}\left(u_{0}\right)=0, \quad u_{0} \in \mathcal{O} \quad \text { and } \quad J\left(u_{0}\right)=\inf _{w \in \mathcal{O}} J(w) .
$$


In [22, Szulkin and Weth have established the existence of ground state solution for problem $\left(P_{1}\right)$ by completing the study made in [16], in the sense that, they also minimize the energy functional on $\mathcal{O}$, however they have used more weaker conditions on $f$, for example $f$ is continuous, $\mathbb{Z}^{N}$-periodic in $x$ and satisfies

$$
|f(x, t)| \leq C\left(1+|t|^{p-1}\right), \quad \forall t \in \mathbb{R} \quad \text { and } \quad x \in \mathbb{R}^{N}
$$

for some $C>0$ and $p \in\left(2,2^{*}\right)$.

$$
\begin{gathered}
f(x, t)=o(t) \text { uniformly in } x \text { as }|t| \rightarrow 0 . \\
F(x, t) /|t|^{2} \rightarrow+\infty \text { uniformly in } x \text { as }|t| \rightarrow+\infty,
\end{gathered}
$$

and

$$
t \mapsto f(x, t) /|t| \text { is strictly increasing on } \mathbb{R} \backslash\{0\} \text {. }
$$

The same approach has been used by Zhang, Xu and Zhang [25, 26] to study a class of indefinite and asymptotically periodic problem.

In 11, Alves and Germano have studied the existence of ground state solution for problem $\left(P_{1}\right)$ by supposing the $f$ has a critical growth for $N \geq 2$, while in [2] the authors have established the existence and concentration of solution for problem $(P)_{\epsilon}$ by supposing that $f$ has a subcritical growth and $V, A$ verify the conditions $(V)$ and $(A)$ respectively.

Motivated by results found [1, 2], in the present paper we intend to study the existence and concentration of solution for problem $(P)_{\epsilon}$ for the case where function $f$ has a critical growth. Since the critical growth brings a lost of compactness, we have established new estimates for the problem. Here, the concentration phenomena is very subtle, because we need to be careful to prove some estimates involving the $L^{\infty}$ norm of the solutions for $\epsilon$ small enough, for more details see Section 2.2 for $N \geq 3$, and Section 3.3 for $N=2$. In additional to conditions $(V)$ and $(A)$ on the functions $V$ and $A$ respectively, we are supposing the following conditions on $f$ :

The Case $N \geq 3$ :

In this case $f: \mathbb{R} \rightarrow \mathbb{R}$ is of the form

$$
f(t)=\xi|t|^{q-1} t+|t|^{2^{*}-2} t, \quad \forall t \in \mathbb{R}
$$

with $\xi>0, q \in\left(2,2^{*}\right)$ and $2^{*}=2 N / N-2$. 
The Case $N=2$ :

In this case $f: \mathbb{R} \rightarrow \mathbb{R}$ is a continuous function that satisfies

$\left(f_{1}\right) \frac{f(t)}{t} \rightarrow 0$ as $t \rightarrow 0$

$\left(f_{2}\right)$ The function $t \mapsto \frac{f(t)}{t}$ is increasing on $(0,+\infty)$ and decreasing on $(-\infty, 0)$;

$\left(f_{3}\right)$ There exists $\theta>2$ such that

$$
0<\theta F(t) \leq f(t) t, \quad \forall t \in \mathbb{R} \backslash\{0\}
$$

where

$$
F(t):=\int_{0}^{t} f(s) d s
$$

$\left(f_{4}\right)$ There exists $\Gamma>0$ such that $|f(t)| \leq \Gamma e^{4 \pi t^{2}}$ for all $t \in \mathbb{R}$;

$\left(f_{5}\right)$ There exist $\tau>0$ and $q>2$ such that $F(t) \geq \tau|t|^{q}$ for all $t \in \mathbb{R}$.

The condition $\left(f_{4}\right)$ says that $f$ can have an exponential critical growth. Here, we recall that a function $f$ has an exponential critical growth, if there is $\alpha_{0}>0$ such that

$$
\lim _{|t| \rightarrow+\infty} \frac{|f(t)|}{e^{\alpha|t|^{2}}}=0, \quad \forall \alpha>\alpha_{0}, \lim _{|t| \rightarrow+\infty} \frac{|f(t)|}{e^{\alpha|t|^{2}}}=+\infty, \quad \forall \alpha<\alpha_{0} .
$$

Our main theorem is the following

Theorem 1.1 Assume $(V),(A),\left(f_{0}\right)$ for $N \geq 3,\left(f_{1}\right)-\left(f_{5}\right)$ for $N=2$. Then, there exist $\tau_{0}, \xi_{0}, \epsilon_{0}>0$ such that $(P)_{\epsilon}$ has a ground state solution $u_{\epsilon}$ for all $\epsilon \in\left(0, \epsilon_{0}\right)$, with $\xi \geq \xi_{0}$ if $N=3$ and $\tau \geq \tau_{0}$ if $N=2$. Moreover, if $x_{\epsilon} \in \mathbb{R}^{N}$ denotes a global maximum point of $\left|u_{\epsilon}\right|$, then

$$
\lim _{\epsilon \rightarrow 0} A\left(\epsilon x_{\epsilon}\right)=\sup _{x \in \mathbb{R}^{N}} A(x) .
$$

In the proof of Theorem 1.1. we will use variational methods to get a critical point for the energy function $I_{\epsilon}: H^{1}\left(\mathbb{R}^{N}\right) \rightarrow \mathbb{R}$ given by

$$
I_{\epsilon}(u)=\frac{1}{2} B(u, u)-\int_{\mathbb{R}^{N}} A(\epsilon x) F(u) d x,
$$


where $B: H^{1}\left(\mathbb{R}^{N}\right) \times H^{1}\left(\mathbb{R}^{N}\right) \rightarrow \mathbb{R}$ is the bilinear form

$$
B(u, v)=\int_{\mathbb{R}^{N}}(\nabla u \nabla v+V(x) u v) d x, \quad \forall u, v \in H^{1}\left(\mathbb{R}^{N}\right) .
$$

It is well known that $I_{\epsilon} \in C^{1}\left(H^{1}\left(\mathbb{R}^{N}\right), \mathbb{R}\right)$ with

$$
I_{\epsilon}^{\prime}(u) v=B(u, v)-\int_{\mathbb{R}^{N}} A(\epsilon x) f(u) v d x, \quad \forall u, v \in H^{1}\left(\mathbb{R}^{N}\right) .
$$

Consequently, critical points of $I_{\epsilon}$ are precisely the weak solutions of $(P)_{\epsilon}$.

Note that the bilinear form $B$ is not positive definite, therefore it does not induce a norm. As in [22], there is an inner product $\langle$,$\rangle in H^{1}\left(\mathbb{R}^{N}\right)$ such that

$$
I_{\epsilon}(u)=\frac{1}{2}\left\|u^{+}\right\|^{2}-\frac{1}{2}\left\|u^{-}\right\|^{2}-\int_{\mathbb{R}^{N}} A(\epsilon x) F(u) d x,
$$

where $\|u\|=\sqrt{\langle u, u\rangle}$ and $H^{1}\left(\mathbb{R}^{N}\right)=E^{+} \oplus E^{-}$corresponds to the spectral decomposition of $-\Delta+V$ with respect to the positive and negative part of the spectrum with $u=u^{+}+u^{-}$, where $u^{+} \in E^{+}$and $u^{-} \in E^{-}$. It is well known that $B$ is positive definite on $E^{+}, B$ is negative definite on $E^{-}$and the norm \|\| is an equivalent norm to the usual norm in $H^{1}\left(\mathbb{R}^{N}\right)$, that is, there are $a, b>0$ such that

$$
b|| u\|\leq\| u\left\|_{H^{1}\left(\mathbb{R}^{N}\right)} \leq a\right\| u \|, \quad \forall u \in H^{1}\left(\mathbb{R}^{N}\right) .
$$

From now on, for each $u \in H^{1}\left(\mathbb{R}^{N}\right), \hat{E}(u)$ designates the set

$$
\hat{E}(u)=E^{-} \oplus[0,+\infty) u .
$$

The plan of the paper is as follows: In Section 2 we will study the existence and concentration of solution for $N \geq 3$, while in Section 3 we will focus our attention to dimension $N=2$.

Notation: In this paper, we use the following notations:

- The usual norms in $H^{1}\left(\mathbb{R}^{N}\right)$ and $L^{p}\left(\mathbb{R}^{N}\right)$ will be denoted by \|\|$_{H^{1}\left(\mathbb{R}^{N}\right)}$ and ||$_{p}$ respectively.

- $C$ denotes (possible different) any positive constant.

- $B_{R}(z)$ denotes the open ball with center $z$ and radius $R$ in $\mathbb{R}^{N}$. 
- We say that $u_{n} \rightarrow u$ in $L_{l o c}^{p}\left(\mathbb{R}^{N}\right)$ when

$$
u_{n} \rightarrow u \quad \text { in } \quad L^{p}\left(B_{R}(0)\right), \quad \forall R>0 .
$$

- If $g$ is a mensurable function, the integral $\int_{\mathbb{R}^{N}} g(x) d x$ will be denoted by $\int g(x) d x$.

- We denote $\delta_{x}$ the Dirac measure.

- If $\varphi \in C_{c}^{\infty}\left(\mathbb{R}^{N}\right)$, the set $\overline{\left\{x \in \mathbb{R}^{N} ; \varphi(x) \neq 0\right\}}$ will be denoted by supp $\varphi$.

\section{The case $N \geq 3$.}

We begin this section by studying the case where $A$ is a constant function. More precisely, we consider the following autonomous problem

$$
\left\{\begin{array}{l}
-\Delta u+V(x) u=\lambda f(u), \quad x \in \mathbb{R}^{N}, \\
u \in H^{1}\left(\mathbb{R}^{N}\right),
\end{array}\right.
$$

with $\lambda \in\left[A_{0},+\infty\right)$ and $f: \mathbb{R} \rightarrow \mathbb{R}$ being of the form

$$
f(t)=\xi|t|^{q-1} t+|t|^{2^{*}-2} t \quad \forall t \in \mathbb{R} ;
$$

with $\xi>0, q \in\left(2,2^{*}\right)$ and $2^{*}=2 N / N-2$.

Associated with $(A P)_{\lambda}$, we have the energy functional $J_{\lambda}: H^{1}\left(\mathbb{R}^{N}\right) \rightarrow \mathbb{R}$ given by

$$
J_{\lambda}(u)=\frac{1}{2} \int\left(|\nabla u|^{2}+V(x)|u|^{2}\right) d x-\lambda \int F(u) d x,
$$

or equivalently

$$
J_{\lambda}(u)=\frac{1}{2}\left\|u^{+}\right\|^{2}-\frac{1}{2}\left\|u^{-}\right\|^{2}-\lambda \int F(u) d x .
$$

In what follows, let us denote by $d_{\lambda}$ the real number defined by

$$
d_{\lambda}=\inf _{u \in \mathcal{N}_{\lambda}} J_{\lambda}(u)
$$

where

$$
\mathcal{N}_{\lambda}=\left\{u \in H^{1}\left(\mathbb{R}^{N}\right) \backslash E^{-} ; J_{\lambda}^{\prime}(u) u=0 \text { and } J_{\lambda}^{\prime}(u) v=0, \forall v \in E^{-}\right\} .
$$


In [1, Alves and Germano have proved that for each $\lambda \in\left[A_{0},+\infty\right)$, the problem $(A P)_{\lambda}$ possesses a ground state solution $u_{\lambda} \in H^{1}\left(\mathbb{R}^{N}\right)$, that is,

$$
u_{\lambda} \in \mathcal{N}_{\lambda}, \quad J_{\lambda}\left(u_{\lambda}\right)=d_{\lambda} \quad \text { and } \quad J_{\lambda}^{\prime}(u)=0 .
$$

A key point to prove the existence of the ground state $u_{\lambda}$ are the following informations involving $d_{\lambda}$ :

$$
0<d_{\lambda}=\inf _{u \in E^{+} \backslash\{0\}} \max _{v \in \widehat{E}(u)} J_{\lambda}(u)
$$

and

$$
d_{\lambda}<\frac{1}{N} \frac{S^{N / 2}}{\lambda^{\frac{N-2}{2}}}, \quad \forall \lambda \geq A_{0} .
$$

Here, we would like to point out that (2.8) holds for $N=3$ if $\xi$ is large enough, while for $N \geq 4$ there is no restriction on $\xi$. This fact justifies why $\xi$ must be large for $N=3$ in Theorem 1.1.

An interesting and important fact is that for each $u \in H^{1}\left(\mathbb{R}^{N}\right) \backslash E^{-}$, $\mathcal{N}_{\lambda} \cap \hat{E}(u)$ is a singleton set and the element of this set is the unique global maximum of $\left.J_{\lambda}\right|_{\hat{E}(u)}$, that is, there are $t^{*} \geq 0$ and $v^{*} \in E^{-}$such that

$$
J_{\lambda}\left(t^{*} u+v^{*}\right)=\max _{w \in \widehat{E}(u)} J_{\lambda}(w) .
$$

After the above commentaries we are ready to prove an important result involving the function $\lambda \mapsto d_{\lambda}$.

Proposition 2.1 The function $\lambda \mapsto d_{\lambda}$ is decreasing and continuous on $\left[A_{0},+\infty\right)$.

Proof. From [2, Proposition 2.3], the function $\lambda \mapsto d_{\lambda}$ is decreasing, and if $\lambda_{1} \leq \lambda_{2} \leq \lambda_{3} \leq \ldots \leq \lambda_{n} \rightarrow \lambda$ then $\lim _{n} d_{\lambda_{n}}=d_{\lambda}$. It suffices to check that $\lambda_{1} \geq \lambda_{2} \geq \lambda_{3} \geq \ldots \geq \lambda_{n} \rightarrow \lambda$ implies $\lim _{n} d_{\lambda_{n}}=d_{\lambda}$. Let $u_{n}$ be a ground state solution of $(A P)_{\lambda_{n}}, t_{n}>0$ and $v_{n} \in E^{-}$verifying

$$
J_{\lambda}\left(t_{n} u_{n}+v_{n}\right)=\max _{\widehat{E}\left(u_{n}\right)} J_{\lambda}
$$

Our goal is to show that $\left(u_{n}\right)$ is bounded in $H^{1}\left(\mathbb{R}^{N}\right)$. First of all, note that

$$
\left(\frac{1}{2}-\frac{1}{q}\right) \int f\left(u_{n}\right) u_{n} d x \leq \int\left(\frac{1}{2} f\left(u_{n}\right) u_{n}-F\left(u_{n}\right)\right) d x=
$$




$$
=\frac{1}{\lambda_{n}}\left(J_{\lambda_{n}}\left(u_{n}\right)-\frac{1}{2} J_{\lambda_{n}}^{\prime}\left(u_{n}\right) u_{n}\right)=\frac{1}{\lambda_{n}} J_{\lambda_{n}}\left(u_{n}\right)=\frac{1}{\lambda_{n}} d_{\lambda_{n}} \leq \frac{1}{\lambda} d_{\lambda},
$$

which proves the boundedness of $\left(\int f\left(u_{n}\right) u_{n} d x\right)$. Fixing $g(t)=$ $\chi_{[-1,1]}(t) f(t)$ and $l(t)=\chi_{[-1,1]^{c}}(t) f(t)$, we have that

$$
g(t)+l(t)=f(t), \quad \forall t \in \mathbb{R} .
$$

From definition of $g$ and $l$, there exists $k>0$ such that

$$
|g(t)|^{r} \leq k t f(t) \text { and }|l(t)|^{s} \leq k t f(t), \quad \forall t \in \mathbb{R},
$$

where $r:=\frac{q+1}{q}$ and $s:=\frac{2^{*}}{2^{*}-1}$. Thus,

$$
\begin{gathered}
\left|\int f\left(u_{n}\right) u_{n}^{+} d x\right| \leq \int\left|g\left(u_{n}\right) u_{n}^{+}\right| d x+\int\left|l\left(u_{n}\right) u_{n}^{+}\right| d x \leq \\
\leq\left(\int\left|g\left(u_{n}\right)\right|^{r} d x\right)^{1 / r}\left|u_{n}^{+}\right|_{q+1}+\left(\int\left|l\left(u_{n}\right)\right|^{s} d x\right)^{1 / s}\left|u_{n}^{+}\right|_{2^{*}} \leq \\
\leq C\left(\int f\left(u_{n}\right) u_{n} d x\right)^{1 / r}\left\|u_{n}^{+}\right\|+C\left(\int f\left(u_{n}\right) u_{n} d x\right)^{1 / s}\left\|u_{n}^{+}\right\| \leq C\left\|u_{n}\right\| .
\end{gathered}
$$

Suppose by contradiction that $\left\|u_{n}\right\| \rightarrow+\infty$. Then

$$
\int \frac{f\left(u_{n}\right) u_{n}^{+}}{\left\|u_{n}\right\|^{2}} d x \rightarrow 0
$$

On the other hand, the equality

$$
0=\frac{J_{\lambda_{n}}^{\prime}\left(u_{n}\right) u_{n}^{+}}{\left\|u_{n}\right\|^{2}}=\frac{\left\|u_{n}^{+}\right\|^{2}}{\left\|u_{n}\right\|^{2}}-\lambda_{n} \int \frac{f\left(u_{n}\right) u_{n}^{+}}{\left\|u_{n}\right\|^{2}} d x
$$

leads to

$$
\frac{\left\|u_{n}^{+}\right\|^{2}}{\left\|u_{n}\right\|^{2}} \rightarrow 0
$$

As $u_{n} \in \mathcal{N}_{\lambda_{n}}$, it follows that $\left\|u_{n}^{-}\right\| \leq\left\|u_{n}^{+}\right\|$, and thus,

$$
1=\frac{\left\|u_{n}^{+}\right\|^{2}}{\left\|u_{n}\right\|^{2}}+\frac{\left\|u_{n}^{-}\right\|^{2}}{\left\|u_{n}\right\|^{2}} \leq 2 \frac{\left\|u_{n}^{+}\right\|^{2}}{\left\|u_{n}\right\|^{2}} \rightarrow 0,
$$


a contradiction. This shows the boundedness of $\left(u_{n}\right)$. We claim that there are $\left(y_{n}\right) \subset \mathbb{Z}^{N}$ and $r, \eta>0$ such that

$$
\int_{B_{r}\left(y_{n}\right)}\left|u_{n}\right|^{2^{*}} d x>\eta, \quad \forall n \in \mathbb{N} .
$$

Arguing by contradiction, if the inequality does not occur, from [19, Lemma 2.1], $u_{n} \rightarrow 0$ in $L^{p}\left(\mathbb{R}^{N}\right)$ for all $p \in\left(2,2^{*}\right]$, and so, $\int f\left(u_{n}\right) u_{n}^{+} d x \rightarrow 0$. This together with the equality below

$$
0=J_{\lambda_{n}}^{\prime}\left(u_{n}\right) u_{n}^{+}=\left\|u_{n}^{+}\right\|^{2}-\lambda_{n} \int f\left(u_{n}\right) u_{n}^{+} d x .
$$

gives $\left\|u_{n}^{+}\right\| \rightarrow 0$, which is a contradiction because $\left\|u_{n}\right\| \geq \sqrt{2 d_{\lambda_{n}}} \geq \sqrt{2 d_{\lambda_{1}}}$. Thereby (2.11) follows.

Define $\widetilde{u}_{n}(x):=u_{n}\left(x+y_{n}\right)$. By [2, Lemma 2.1], $\widetilde{u}_{n}^{+}(x)=u_{n}^{+}\left(x+y_{n}\right)$ and $\left(\widetilde{u}_{n}\right)$ is bounded in $H^{1}\left(\mathbb{R}^{N}\right)$. In the sequel, let us assume that for some subsequence $\widetilde{u}_{n} \rightarrow u$ in $H^{1}\left(\mathbb{R}^{N}\right)$. Our goal is to show that $u \neq 0$. Inspired by [1. Lemma 2.17], let us suppose by contradiction $u=0$ and

$$
\left|\nabla \widetilde{u}_{n}\right|^{2} \rightarrow \mu, \quad\left|\widetilde{u}_{n}\right|^{2^{*}} \rightarrow \nu \text { in } \mathcal{M}^{+}\left(\mathbb{R}^{N}\right) .
$$

By Concentration-Compactness Principle due to Lions 14, there exist a countable set $\mathrm{J},\left(x_{i}\right)_{i \in \mathrm{J}} \subset \mathbb{R}^{N}$ and $\left(\mu_{i}\right)_{i \in \mathrm{J}},\left(\nu_{i}\right)_{i \in \mathrm{J}} \subset[0,+\infty)$ such that

$$
\nu=\sum_{i \in \mathrm{J}} \nu_{i} \delta_{x_{i}}, \mu \geq \sum_{i \in \mathrm{J}} \mu_{i} \delta_{x_{i}}, \text { and } \mu_{i}=S \nu_{i}^{2 / 2^{*}} .
$$

We will prove that $\nu_{i}=0$ for all $i \in J$. Suppose there exists $i \in J$ such that $\nu_{i} \neq 0$. Then,

$$
\begin{aligned}
d_{\lambda} & \geq \lim _{n} d_{\lambda_{n}}=\lim _{n}\left(J_{\lambda_{n}}\left(u_{n}\right)-\frac{1}{2} J_{\lambda_{n}}^{\prime}\left(u_{n}\right) u_{n}\right) \\
& \geq \lim _{n} \lambda_{n}\left(\frac{1}{2}-\frac{1}{2^{*}}\right) \int\left|u_{n}\right|^{2^{*}} d x \\
& =\lim _{n} \frac{\lambda_{n}}{N} \int\left|\widetilde{u}_{n}\right|^{2^{*}} d x=\frac{\lambda}{N} \sum_{j \in \mathrm{J}} \nu_{j},
\end{aligned}
$$

which means

$$
d_{\lambda} \geq \frac{\lambda}{N} \sum_{j \in \mathrm{J}} \nu_{j}
$$


Let $\varphi_{\delta}(x):=\varphi\left(\frac{x-x_{i}}{\delta}\right)$ for all $x \in \mathbb{R}^{N}$ and $\delta>0$, where $\varphi \in C_{c}^{\infty}\left(\mathbb{R}^{N}\right)$ is such that $\varphi \equiv 1$ on $B_{1}(0), \varphi \equiv 0$ on $\mathbb{R}^{N} \backslash B_{2}(0), 0 \leq \varphi \leq 1$ and $|\nabla \varphi| \leq 2$. Consequently $\left(\varphi_{\delta} \widetilde{u}_{n}\right)$ is bounded in $H^{1}\left(\mathbb{R}^{N}\right)$ and

$$
J_{\lambda_{n}}^{\prime}\left(\widetilde{u}_{n}\right)\left(\varphi_{\delta} \widetilde{u}_{n}\right)=0
$$

that is,

$$
\int \nabla \widetilde{u}_{n} \nabla\left(\varphi_{\delta} \widetilde{u}_{n}\right) d x+\int V(x) \varphi_{\delta} \widetilde{u}_{n}^{2} d x=\lambda_{n} \xi \int\left|\widetilde{u}_{n}\right|^{q+1} \varphi_{\delta} d x+\lambda_{n} \int\left|\widetilde{u}_{n}\right|^{2^{*}} \varphi_{\delta} d x .
$$

Passing to the limit as $n \rightarrow+\infty$,

$$
\int \varphi_{\delta} d \mu=\lambda \int \varphi_{\delta} d \nu
$$

Now, taking the limit $\delta \rightarrow 0$,

$$
\mu\left(x_{i}\right)=\lambda \nu_{i}
$$

From the fact that $\mu\left(x_{i}\right) \geq \mu_{i}$, we derive

$$
S \nu_{i}^{2 / 2^{*}}=\mu_{i} \leq \mu\left(x_{i}\right)=\lambda \nu_{i}
$$

and so

$$
S^{N / 2} \leq \lambda^{N / 2} \nu_{i}
$$

Consequently,

$$
\frac{\lambda}{N} \nu_{i} \geq \frac{1}{N} \frac{S^{N / 2}}{\lambda^{\frac{N-2}{2}}}
$$

From (2.12) and (2.13),

$$
d_{\lambda} \geq \frac{1}{N} \frac{S^{N / 2}}{\lambda^{\frac{N-2}{2}}}
$$

contrary to (2.8). From this, $\nu_{i}=0$ for all $i \in \mathrm{J}$ and $\widetilde{u}_{n} \rightarrow 0$ in $L_{l o c}^{2^{*}}\left(\mathbb{R}^{N}\right)$, which contradicts (2.11). This permit us to conclude that $u \neq 0$.

Claim 2.2 If $u^{+}=0$, then $u^{-}=0$.

In fact, if $u^{+}=0$,

$$
\int f(u) u^{-} d x=\int f(u) u^{+} d x+\int f(u) u^{-} d x=\int f(u) u d x \geq 0 .
$$


On the other hand, letting $n \rightarrow+\infty$ in the equality below

$$
0=J_{\lambda_{n}}\left(\widetilde{u}_{n}\right) u^{-}=B\left(\widetilde{u}_{n}, u^{-}\right)-\lambda_{n} \int f\left(\widetilde{u}_{n}\right) u^{-} d x
$$

we find

$$
-\left\|u^{-}\right\|^{2}=B\left(u, u^{-}\right)=\lambda \int f(u) u^{-} d x \geq 0,
$$

thereby showing that $u^{-}=0$.

The Claim 2.2 implies that $u^{+} \neq 0$, because $u \neq 0$ and $u=u^{+}+u^{-}$. Define $\mathcal{V}:=\left\{\widetilde{u}_{n}^{+}\right\}_{n \in \mathbb{N}}$. Since $\widetilde{u}_{n}^{+} \rightarrow u^{+} \neq 0$, then $0 \notin \overline{\mathcal{V}}^{\sigma\left(H^{1}\left(\mathbb{R}^{N}\right), H^{1}\left(\mathbb{R}^{N}\right)^{\prime}\right)}$ and $\mathcal{V}$ is bounded in $H^{1}\left(\mathbb{R}^{N}\right)$. Applying [2, Lemma 2.2], there exists $R>0$ such that

$$
J_{\lambda} \leq 0 \text { on } \widehat{E}(u) \backslash B_{R}(0), \text { for all } u \in \mathcal{V} .
$$

Setting $\widetilde{v}_{n}(x):=v_{n}\left(x+y_{n}\right)$,

$$
J_{\lambda}\left(t_{n} \widetilde{u}_{n}+\widetilde{v}_{n}\right)=J_{\lambda}\left(t_{n} u_{n}+v_{n}\right) \geq d_{\lambda}>0 .
$$

By (2.14) and (2.15), $\left\|t_{n} \widetilde{u}_{n}+\widetilde{v}_{n}\right\| \leq R$ for all $n \in \mathbb{N}$. As $\left\|t_{n} u_{n}+v_{n}\right\|=$ $\left\|t_{n} \widetilde{u}_{n}+\widetilde{v}_{n}\right\|,\left(t_{n} u_{n}+v_{n}\right)$ is also bounded in $H^{1}\left(\mathbb{R}^{N}\right)$ and

$$
\begin{aligned}
d_{\lambda} & \leq J_{\lambda}\left(t_{n} u_{n}+v_{n}\right)=\left(\lambda_{n}-\lambda\right) \int F\left(t_{n} u_{n}+v_{n}\right) d x+J_{\lambda_{n}}\left(t_{n} u_{n}+v_{n}\right) \leq \\
& \leq o_{n}+J_{\lambda_{n}}\left(u_{n}\right)=o_{n}+d_{\lambda_{n}} \leq o_{n}+d_{\lambda},
\end{aligned}
$$

from where it follows that $\lim _{n} d_{\lambda_{n}}=d_{\lambda}$.

\subsection{Existence of ground state for problem $(P)_{\epsilon}$.}

In the sequel, we fix

$$
\mathcal{M}_{\epsilon}:=\left\{u \in H^{1}\left(\mathbb{R}^{N}\right) \backslash E^{-} ; I_{\epsilon}^{\prime}(u) u=I_{\epsilon}^{\prime}(u) v=0, \text { for all } v \in E^{-}\right\}
$$

and

$$
c_{\epsilon}=\inf _{\mathcal{M}_{\epsilon}} I_{\epsilon} .
$$

By using the same arguments found in [1], it follows that $c_{\epsilon}>0$, and for each $u \in H^{1}\left(\mathbb{R}^{N}\right) \backslash E^{-}$, there exist $t \geq 0$ and $v \in E^{-}$verifying

$$
I_{\epsilon}(t u+v)=\max _{\widehat{E}(u)} I_{\epsilon} \text { and }\{t u+v\}=\mathcal{M}_{\epsilon} \cap \widehat{E}(u) .
$$


The same idea of [1, Lemma 2.6] proves that

$$
\left\|u^{+}\right\|^{2} \geq 2 c_{\epsilon}, \quad \text { for all } u \in \mathcal{M}_{\epsilon} \quad \text { and } \quad \epsilon>0 .
$$

In what follows, without loss of generality we assume that

$$
A(0)=\max _{x \in \mathbb{R}^{N}} A(x) .
$$

Our first result in this section establishes an important relation involving the levels $c_{\epsilon}$ and $c_{0}$.

Lemma 2.3 The limit $\lim _{\epsilon \rightarrow 0} c_{\epsilon}=c_{0}$ holds. Moreover, let $w_{0}$ be a ground state solution of the problem $(P)_{0}, t_{\epsilon} \geq 0$ and $v_{\epsilon} \in E^{-}$such that $t_{\epsilon} w_{0}+v_{\epsilon} \in \mathcal{M}_{\epsilon}$. Then

$$
t_{\epsilon} \rightarrow 1 \text { and } v_{\epsilon} \rightarrow 0 \quad \text { as } \epsilon \rightarrow 0
$$

Proof. See [2, Lemmas 3.1 and 3.3].

Corollary 2.4 There exists $\epsilon_{0}>0$ such that

$$
c_{\epsilon}<d_{A_{\infty}} \quad \text { and } \quad c_{\epsilon}<\frac{S^{N / 2}}{N A(0)^{\frac{N-2}{2}}}, \quad \forall \epsilon \in\left(0, \epsilon_{0}\right) .
$$

Proof. Since $c_{0}<d_{A_{\infty}}$ and

$$
c_{0}<\frac{S^{N / 2}}{N A(0)^{\frac{N-2}{2}}} \quad(\text { see }(2.8)),
$$

the corollary is an immediate consequence of Lemma 2.3.

The next result is essential to show the existence of ground state solution of $(P)_{\epsilon}$ for $\epsilon$ small enough. Since it follows as in [1, Proposition 2.16], we omit its proof.

Proposition 2.5 There exists a bounded sequence $\left(u_{n}\right) \subset \mathcal{M}_{\epsilon}$ such that $\left(u_{n}\right)$ is $(P S)_{c_{\epsilon}}$ for $I_{\epsilon}$.

The following result is the main result this section

Theorem 2.6 The problem $(P)_{\epsilon}$ has a ground state solution for all $\epsilon \epsilon$ $\left(0, \epsilon_{0}\right)$, where $\epsilon_{0}>0$ was given in Corollary 2.4. 
Proof. Let $\left(u_{n}\right) \subset \mathcal{M}_{\epsilon}$ be the $(P S)_{c_{\epsilon}}$ sequence for $I_{\epsilon}$ given in Proposition 2.5. Then, there exist $\left(z_{n}\right) \subset \mathbb{Z}^{N}$ and $\eta, r>0$ such that

$$
\int_{B_{r}\left(z_{n}\right)}\left|u_{n}\right|^{2^{*}} d x>\eta, \quad \forall n \in \mathbb{N} .
$$

In fact, otherwise, by [19, Lemma 2.1], $u_{n} \rightarrow 0$ in $L^{p}\left(\mathbb{R}^{N}\right)$ for all $p \in\left(2,2^{*}\right]$. Then,

$$
\left\|u_{n}^{+}\right\|^{2}=\int A(\epsilon x) f\left(u_{n}\right) u_{n}^{+} d x \rightarrow 0,
$$

which is a contradiction with (2.16), and (2.17) is proved.

Claim 2.7 The sequence $\left(z_{n}\right)$ is bounded in $\mathbb{R}^{N}$.

Arguing by contradiction, suppose $\left|z_{n}\right| \rightarrow+\infty$ and define $w_{n}(x):=u_{n}(x+$ $\left.z_{n}\right)$. Then $\left(w_{n}\right)$ is bounded, and for some subsequence, $w_{n} \rightarrow w$ in $H^{1}\left(\mathbb{R}^{N}\right)$. Our goal is to prove that $w \neq 0$. Suppose $w=0$ and

$$
\left|\nabla w_{n}\right|^{2} \rightarrow \mu, \quad\left|w_{n}\right|^{2^{*}} \rightarrow \nu, \quad \text { in } \mathcal{M}^{+}\left(\mathbb{R}^{N}\right) .
$$

By Concentration-Compactness Principle due to Lions [14, there exist a countable set $\mathrm{J},\left(x_{i}\right)_{i \in \mathrm{J}} \subset \mathbb{R}^{N}$ and $\left(\mu_{i}\right)_{i \in \mathrm{J}},\left(\nu_{i}\right)_{i \in \mathrm{J}} \subset[0,+\infty)$ satisfying

$$
\nu=\sum_{i \in \mathrm{J}} \nu_{i} \delta_{x_{i}}, \quad \mu \geq \sum_{i \in \mathrm{J}} \mu_{i} \delta_{x_{i}}, \quad \text { and } \quad \mu_{i}=S \nu_{i}^{2 / 2^{*}} .
$$

Next, we are going to prove that $\nu_{i}=0$ for all $i \in J$. Suppose that there exists $i \in \mathrm{J}$ such that $\nu_{i} \neq 0$. Note that

$$
\begin{aligned}
& c_{\epsilon}=\lim _{n}\left(I_{\epsilon}\left(u_{n}\right)-\frac{1}{2} I_{\epsilon}^{\prime}\left(u_{n}\right) u_{n}\right) \geq \frac{1}{N} \lim _{n} \int A(\epsilon x)\left|u_{n}\right|^{2^{*}} d x= \\
& =\frac{1}{N} \lim _{n} \int A\left(\epsilon x+\epsilon z_{n}\right)\left|w_{n}\right|^{2^{*}} d x \geq \frac{1}{N} \lim _{n} \int_{B_{\delta}\left(x_{i}\right)} A\left(\epsilon x+\epsilon z_{n}\right)\left|w_{n}\right|^{2^{*}} d x= \\
& =\frac{1}{N} \lim _{n} \int_{B_{\delta}\left(x_{i}\right)}\left(A\left(\epsilon x+\epsilon z_{n}\right)-A_{\infty}\right)\left|w_{n}\right|^{2^{*}} d x+\frac{1}{N} \lim _{n} \int_{B_{\delta}\left(x_{i}\right)} A_{\infty}\left|w_{n}\right|^{2^{*}} d x \geq \\
& \geq \frac{1}{N} \int A_{\infty} \varphi_{\delta / 2}(x) d \nu
\end{aligned}
$$

where $\varphi_{\delta}(x)=\varphi\left(\frac{x-x_{i}}{\delta}\right)$, and $\varphi \in C_{c}^{\infty}\left(\mathbb{R}^{N}\right)$ satisfies $0 \leq \varphi \leq 1,|\nabla \varphi| \leq 2$, $\varphi \equiv 1$ on $B_{1}(0)$ and $\varphi \equiv 0$ on $\mathbb{R}^{N} \backslash B_{2}(0)$.

By Dominated Convergence Theorem,

$$
\lim _{\delta \rightarrow 0} \int A_{\infty} \varphi_{\delta / 2}(x) d \nu=A_{\infty} \nu_{i}
$$


thus

$$
c_{\epsilon} \geq \frac{1}{N} A_{\infty} \nu_{i}
$$

On the other hand, by a simple calculus, $\left(\varphi_{\delta} w_{n}\right)$ is bounded in $H^{1}\left(\mathbb{R}^{N}\right)$. Setting $\varphi_{\delta, n}(x):=\varphi_{t}\left(x-z_{n}\right)$,

$$
\left\|\varphi_{\delta, n} u_{n}\right\|=\left\|\varphi_{\delta} w_{n}\right\|, \quad \forall n \in \mathbb{N}
$$

and so,

$$
I_{\epsilon}^{\prime}\left(u_{n}\right)\left(\varphi_{\delta, n} u_{n}\right) \rightarrow 0
$$

or equivalently

$$
\begin{aligned}
& \int\left|\nabla w_{n}\right|^{2} \varphi_{\delta} d x+\int\left(\nabla w_{n} \nabla \varphi_{\delta}\right) w_{n} d x+\int V(x) \varphi_{\delta} w_{n}^{2} d x- \\
& -\int A\left(\epsilon x+\epsilon z_{n}\right)\left|w_{n}\right|^{q+1} \varphi_{\delta} d x-\int A\left(\epsilon x+\epsilon z_{n}\right)\left|w_{n}\right|^{2^{*}} \varphi_{\delta} d x \rightarrow 0
\end{aligned}
$$

Taking the limit $n \rightarrow+\infty$, and after $\delta \rightarrow 0$, we obtain

$$
\mu\left(x_{i}\right)=A_{\infty} \nu_{i}
$$

Since $S \nu_{i}^{2 / 2^{*}} \leq \mu\left(x_{i}\right)$, it follows that

$$
S^{N / 2} \leq A_{\infty}^{\frac{N}{2}} \nu_{i} \leq A(0)^{\frac{N-2}{2}} A_{\infty} \nu_{i} .
$$

By (2.18) and (2.19),

$$
c_{\epsilon} \geq \frac{S^{N / 2}}{N A(0)^{\frac{N-2}{2}}},
$$

which is impossible by Corollary 2.4. Consequently $\nu_{i}=0$ for all $i \in \mathrm{J}$, which means $w_{n} \rightarrow 0$ in $L_{l o c}^{2^{*}}\left(\mathbb{R}^{N}\right)$, contrary to (2.17). From this, $w \neq 0$.

Now, consider $\psi \in H^{1}\left(\mathbb{R}^{N}\right)$ and $\psi_{n}(x):=\psi\left(x+z_{n}\right)$. Then,

$$
o_{n}(1)=I_{\epsilon}^{\prime}\left(u_{n}\right) \psi_{n}=B\left(u_{n}, \psi_{n}\right)-\int A(\epsilon x) f\left(u_{n}\right) \psi_{n} d x
$$

or equivalently

$$
o_{n}=B\left(w_{n}, \psi\right)-\int A\left(\epsilon x+\epsilon z_{n}\right) f\left(w_{n}\right) \psi d x .
$$


Taking the limit $n \rightarrow+\infty, J_{A_{\infty}}^{\prime}(w) \psi=0$. As $\psi \in H^{1}\left(\mathbb{R}^{N}\right)$ is arbitrary, $w$ is a critical point of $J_{A_{\infty}}$, and thus, by Fatou's Lemma

$$
\begin{aligned}
d_{A_{\infty}} & \leq J_{A_{\infty}}(w)=J_{A_{\infty}}(w)-\frac{1}{2} J_{A_{\infty}}^{\prime}(w) w \\
& =\int A_{\infty}\left(\frac{1}{2} f(w) w-F(w)\right) d x \\
& \leq \liminf _{n} \int A\left(\epsilon x+\epsilon z_{n}\right)\left(\frac{1}{2} f\left(w_{n}\right) w_{n}-F\left(x, w_{n}\right)\right) d x \\
& =\liminf _{n} \int A(\epsilon x)\left(\frac{1}{2} f\left(u_{n}\right) u_{n}-F\left(u_{n}\right)\right) d x \\
& =\lim _{n}\left(I_{\epsilon}\left(u_{n}\right)-\frac{1}{2} I_{\epsilon}^{\prime}\left(u_{n}\right) u_{n}\right)=c_{\epsilon}<d_{A_{\infty}}
\end{aligned}
$$

which is absurd. Thereby $\left(z_{n}\right)$ is bounded in $\mathbb{R}^{N}$, and the claim follows.

Consider $R>0$ such that $B_{r}\left(z_{n}\right) \subset B_{R}(0)$. By (2.17),

$$
\int_{B_{R}(0)}\left|u_{n}\right|^{2^{*}} d x>\eta, \quad \forall n \in \mathbb{N} \text {. }
$$

By considering that $u_{n} \rightarrow u$ and proceeding as in Claim 2.7. $u \neq 0$. Since $u$ is a nontrivial critical point for $I_{\epsilon}$, we must have $I_{\epsilon}(u) \geq c_{\epsilon}$. On the other hand, by Fatou's Lemma,

$$
\begin{aligned}
c_{\epsilon} & =\lim _{n}\left(I_{\epsilon}\left(u_{n}\right)-\frac{1}{2} I_{\epsilon}^{\prime}\left(u_{n}\right) u_{n}\right)=\lim _{n} \int A(\epsilon x)\left(\frac{1}{2} f\left(u_{n}\right) u_{n}-F\left(u_{n}\right)\right) d x \\
& \geq \int A(\epsilon x)\left(\frac{1}{2} f(u) u-F(u)\right) d x=I_{\epsilon}(u)-\frac{1}{2} I_{\epsilon}^{\prime}(u) u=I_{\epsilon}(u) .
\end{aligned}
$$

This proves that $u$ is a ground state solution of $(P)_{\epsilon}$ for all $\epsilon \in\left(0, \epsilon_{0}\right)$.

\subsection{Concentration of the solutions.}

In what follows, we consider the set

$$
\mathcal{A}:=\left\{z \in \mathbb{R}^{N} ; A(z)=A(0)\right\},
$$

and a sequence $\left(\epsilon_{n}\right) \subset\left(0, \epsilon_{0}\right)$ with $\epsilon_{n} \rightarrow 0$ as $n \rightarrow+\infty$. Moreover, we fix $u_{n} \in H^{1}\left(\mathbb{R}^{N}\right)$ satisfying

$$
I_{n}\left(u_{n}\right)=c_{n} \quad \text { and } \quad I_{n}^{\prime}\left(u_{n}\right)=0,
$$

where $I_{n}:=I_{\epsilon_{n}}$ and $c_{n}:=c_{\epsilon_{n}}$. Using the same arguments explored in [1, Lemma 2.6],

$$
\left\|u_{n}^{+}\right\|^{2} \geq 2 c_{n} \geq 2 c_{0}, \quad \forall n \in \mathbb{N} .
$$


Lemma 2.8 The sequence $\left(u_{n}\right)$ is bounded in $H^{1}\left(\mathbb{R}^{N}\right)$.

Proof. See [1, Lemma 2.10].

Lemma 2.9 There exist $\left(y_{n}\right) \subset \mathbb{Z}^{N}$ and $r, \eta>0$ such that

$$
\int_{B_{r}\left(y_{n}\right)}\left|u_{n}\right|^{2^{*}} d x>\eta, \quad \forall n \in \mathbb{N}
$$

Proof. Suppose the lemma were false. Then, by [19, Lemma 2.1], $u_{n} \rightarrow 0$ in $L^{p}\left(\mathbb{R}^{N}\right)$ for all $p \in\left(2,2^{*}\right]$, and so,

$$
\int A\left(\epsilon_{n} x\right) f\left(u_{n}\right) u_{n}^{+} d x \rightarrow 0 .
$$

As $I_{n}^{\prime}\left(u_{n}\right) u_{n}^{+}=0$, it follows that $\left\|u_{n}^{+}\right\|^{2} \rightarrow 0$, a contradiction. This proves the lemma.

In the sequel, we fix $v_{n}(x):=u_{n}\left(x+y_{n}\right)$ for all $x \in \mathbb{R}^{N}$ and for all $n \in \mathbb{N}$. Thereby, for some subsequence, we can assume that $v_{n} \rightarrow v$ in $H^{1}\left(\mathbb{R}^{2}\right)$. It is very important to point out that only one of the cases below holds for some subsequence:

$$
\epsilon_{n} y_{n} \rightarrow z \in \mathbb{R}^{N}
$$

or

$$
\left|\epsilon_{n} y_{n}\right| \rightarrow+\infty
$$

For this reason, we will consider a subsequence of $\left(\epsilon_{n}\right)$ such that one of the above conditions holds. Have this in mind, let us denote

$$
A_{z}:=\left\{\begin{array}{l}
A(z), \text { if the condition (1) holds } \\
A_{\infty}, \text { if the condition (2) holds. }
\end{array}\right.
$$

Since $A$ is continuous, it follows that $\left|A\left(\epsilon_{n} x+\epsilon_{n} y_{n}\right)-A_{z}\right| \rightarrow 0$ uniformly with respect to $x$ on bounded Borel sets $B \subset \mathbb{R}^{N}$. Consequently

$$
\lim \int_{B} A\left(\epsilon_{n} x+\epsilon_{n} y_{n}\right)\left|v_{n}\right|^{2^{*}} \varphi d x=\lim \int_{B} A_{z}\left|v_{n}\right|^{2^{*}} \varphi d x,
$$

for each $\varphi \in L^{\infty}\left(\mathbb{R}^{N}\right)$.

By using (2.21) and applying the same idea of Claim 2.7, we see that $v \neq 0$. 
Lemma 2.10 The sequence $\left(\epsilon_{n} y_{n}\right)$ is bounded in $\mathbb{R}^{N}$. Moreover, $J_{A(0)}^{\prime}(v)=$ 0 and if $\epsilon_{n} y_{n} \rightarrow z \in \mathbb{R}^{N}$, then $z \in \mathcal{A}$.

Proof. First of all, we will prove that $\left(\epsilon_{n} y_{n}\right)$ is bounded. Suppose that $\left|\epsilon_{n} y_{n}\right| \rightarrow+\infty$. Consider $\psi \in C_{c}^{\infty}\left(\mathbb{R}^{N}\right)$ and $\psi_{n}(x):=\psi\left(x-y_{n}\right)$. Since $I_{n}^{\prime}\left(u_{n}\right) \psi_{n}=0$ for all $n \in \mathbb{N}$, then

$$
\int \nabla u_{n} \nabla \psi_{n}+V(x) u_{n} \psi_{n} d x=\int A\left(\epsilon_{n} x\right) f\left(u_{n}\right) \psi_{n} d x
$$

or equivalently

$$
\int \nabla v_{n} \nabla \psi+V(x) v_{n} \psi d x=\int A\left(\epsilon_{n} x+\epsilon_{n} y_{n}\right) f\left(v_{n}\right) \psi d x .
$$

Taking the limit $n \rightarrow+\infty$, we derive

$$
\int \nabla v \nabla \psi+V(x) v \psi d x=\int A_{\infty} f(v) \psi d x
$$

thereby showing that $J_{A_{\infty}}^{\prime}(v)=0$. As $v \neq 0$, the Fatou's Lemma yields

$$
\begin{aligned}
d_{A_{\infty}} & \leq J_{A_{\infty}}(v)=J_{A_{\infty}}(v)-\frac{1}{2} J_{A_{\infty}}^{\prime}(v) v=\int A_{\infty}\left(\frac{1}{2} f(v) v-F(v)\right) d x \\
& \leq \liminf _{n} \int A\left(\epsilon_{n} x+\epsilon_{n} y_{n}\right)\left(\frac{1}{2} f\left(v_{n}\right) v_{n}-F\left(v_{n}\right)\right) d x \\
& =\liminf _{n} \int A\left(\epsilon_{n} x\right)\left(\frac{1}{2} f\left(u_{n}\right) u_{n}-F\left(u_{n}\right)\right) d x \\
& =\liminf _{n}\left(I_{n}\left(u_{n}\right)-\frac{1}{2} I_{n}^{\prime}\left(u_{n}\right) u_{n}\right)=\lim _{n} c_{n}=c_{0},
\end{aligned}
$$

which is absurd, because $c_{0}<d_{A_{\infty}}$. This completes the proof that $\left(\epsilon_{n} y_{n}\right)$ is bounded in $\mathbb{R}^{N}$. Now suppose $\epsilon_{n} y_{n} \rightarrow z \in \mathbb{R}^{N}$. Arguing as above,

$$
\int \nabla v \nabla \psi+V(x) v \psi d x=\int A(z) f(v) \psi d x, \quad \psi \in C_{c}^{\infty}\left(\mathbb{R}^{N}\right),
$$

and so, $J_{A(z)}^{\prime}(v)=0$. Hence,

$d_{A(z)} \leq J_{A(z)}(v)-\frac{1}{2} J_{A(z)}^{\prime}(v) v \leq \liminf _{n}\left(I_{n}\left(u_{n}\right)-\frac{1}{2} I_{n}^{\prime}\left(u_{n}\right) u_{n}\right)=c_{0}=d_{A(0)}$.

Since $\lambda \mapsto d_{\lambda}$ is decreasing and $d_{A(z)} \leq d_{A(0)}$, we must have $A(0) \leq$ $A(z)$. From the fact that $A(0)=\max _{x \in \mathbb{R}^{N}} A(x)$, we obtain $A(0)=A(z)$, or equivalently, $z \in \mathcal{A}$. Moreover, we also have $J_{A(0)}^{\prime}(v)=J_{A(z)}^{\prime}(v)=0$. 
From now on we consider $\epsilon_{n} y_{n} \rightarrow z$ with $z \in \mathcal{A}$. Our goal is to prove that $v_{n} \rightarrow v$ in $H^{1}\left(\mathbb{R}^{N}\right)$ and $v_{n}(x) \rightarrow 0$ as $|x| \rightarrow+\infty$ uniformly in $n$. Have this in mind, we need of the following estimate

Proposition 2.11 There exists $h \in L^{1}\left(\mathbb{R}^{N}\right)$ and a subsequence of $\left(v_{n}\right)$ such that

$$
\left|f\left(v_{n}(x)\right) v_{n}(x)\right| \leq h(x), \quad \forall x \in \mathbb{R}^{N} \quad \text { and } \quad n \in \mathbb{N} .
$$

Proof. By Fatou's Lemma,

$$
\begin{aligned}
d_{A(0)} & \leq J_{A(0)}(v)=J_{A(0)}(v)-\frac{1}{2} J_{A(0)}^{\prime}(v) v \\
& =\int A(0)\left(\frac{1}{2} f(v) v-F(v)\right) d x \\
& =\int A(z)\left(\frac{1}{2} f(v) v-F(v)\right) d x \\
& \leq \liminf _{n} \int A\left(\epsilon_{n} x+\epsilon_{n} y_{n}\right)\left(\frac{1}{2} f\left(v_{n}\right) v_{n}-F\left(v_{n}\right)\right) d x \\
& \leq \limsup _{n} \int A\left(\epsilon_{n} x+\epsilon_{n} y_{n}\right)\left(\frac{1}{2} f\left(v_{n}\right) v_{n}-F\left(v_{n}\right)\right) d x \\
& =\limsup _{n} \int A\left(\epsilon_{n} x\right)\left(\frac{1}{2} f\left(u_{n}\right) u_{n}-F\left(u_{n}\right)\right) d x \\
& =\limsup _{n}\left(I_{n}\left(u_{n}\right)-\frac{1}{2} I_{n}^{\prime}\left(u_{n}\right) u_{n}\right)=\lim _{n} c_{n}=c_{0}=d_{A(0)},
\end{aligned}
$$

from where it follows that

$\lim _{n} \int A\left(\epsilon_{n} x+\epsilon_{n} y_{n}\right)\left(\frac{1}{2} f\left(v_{n}\right) v_{n}-F\left(v_{n}\right)\right) d x=\int A(z)\left(\frac{1}{2} f(v) v-F(v)\right) d x$

Since

$$
A\left(\epsilon_{n} x+\epsilon_{n} y_{n}\right)\left(\frac{1}{2} f\left(v_{n}\right) v_{n}-F\left(v_{n}\right)\right) \geq 0
$$

and

$A\left(\epsilon_{n} x+\epsilon_{n} y_{n}\right)\left(\frac{1}{2} f\left(v_{n}\right) v_{n}-F\left(v_{n}\right)\right) \rightarrow A(z)\left(\frac{1}{2} f(v) v-F(v)\right)$ a.e. in $\mathbb{R}^{N}$,

we can ensure that

$A\left(\epsilon_{n} x+\epsilon_{n} y_{n}\right)\left(\frac{1}{2} f\left(v_{n}\right) v_{n}-F\left(v_{n}\right)\right) \rightarrow A(z)\left(\frac{1}{2} f(v) v-F(v)\right) \quad$ in $\quad L^{1}\left(\mathbb{R}^{N}\right)$. 
Thereby, there exists $\widetilde{h} \in L^{1}\left(\mathbb{R}^{N}\right)$ such that, for some subsequence,

$$
A\left(\epsilon_{n} x+\epsilon_{n} y_{n}\right)\left(\frac{1}{2} f\left(v_{n}\right) v_{n}-F\left(v_{n}\right)\right) \leq \widetilde{h}(x), \quad \forall n \in \mathbb{N} .
$$

As

$$
\left(\frac{1}{2}-\frac{1}{q+1}\right)\left(\inf _{\mathbb{R}^{N}} A\right) f\left(v_{n}\right) v_{n} \leq A\left(\epsilon_{n} x+\epsilon_{n} y_{n}\right)\left(\frac{1}{2} f\left(v_{n}\right) v_{n}-F\left(v_{n}\right)\right),
$$

we get the desired result.

An immediate consequence of the last proposition is the following corollary

Corollary 2.12 $v_{n} \rightarrow v$ in $L^{2^{*}}\left(\mathbb{R}^{N}\right)$.

Proof. The result follows because $\left|v_{n}\right|^{2^{*}} \leq f\left(v_{n}\right) v_{n}$ for all $n \in \mathbb{N}$ and $v_{n}(x) \rightarrow v(x)$ a.e. in $\mathbb{R}^{N}$.

Our next result establishes a key estimate involving the $L^{\infty}$ norm on balls for the sequence $\left(v_{n}\right)$. To this end, we fix $v_{n,+}=\max \left\{0, v_{n}\right\}$ and $v_{n,-}=\max \left\{0,-v_{n}\right\}$.

Lemma 2.13 There exist $R>0$ and $C>0$ such that

$$
\left|v_{n}\right|_{L^{\infty}\left(B_{R}(x)\right)} \leq C\left|v_{n}\right|_{L^{2^{*}}\left(B_{2 R}(x)\right)}, \quad \forall n \in \mathbb{N} \quad \text { and } \quad \forall x \in \mathbb{R}^{N}
$$

Hence, as $\left(v_{n}\right)$ is a bounded sequence in $L^{2^{*}}\left(\mathbb{R}^{N}\right), v_{n} \in L^{\infty}\left(\mathbb{R}^{N}\right)$ and there is $C>0$ such that

$$
\left|v_{n}\right|_{\infty} \leq C, \quad \forall n \in \mathbb{N} .
$$

Proof. It suffices to check that

$$
\left|v_{n,+}\right|_{L^{\infty}\left(B_{R}(x)\right)} \leq C\left|v_{n,+}\right|_{L^{2^{*}}\left(B_{2 R}(x)\right)},
$$

for all $n \in \mathbb{N}$ and $x \in \mathbb{R}^{N}$, because similar reasoning proves

$$
\left|v_{n,-}\right|_{L^{\infty}\left(B_{R}(x)\right)} \leq C\left|v_{n,-}\right|_{L^{2^{*}}\left(B_{2 R}(x)\right)},
$$

for all $n \in \mathbb{N}$ and $x \in \mathbb{R}^{N}$. To begin with, we recall that there exist $c_{1}, c_{2}>0$ satisfying

$$
|f(t)| \leq c_{1}|t|+c_{2}|t|^{2^{*}-1}, \quad \text { for all } t \in \mathbb{R}
$$


and that $v_{n}$ is a solution for the problem

$$
\left\{\begin{array}{l}
-\Delta v_{n}+V(x) v_{n}=A\left(\epsilon_{n} x+\epsilon_{n} y_{n}\right) f\left(v_{n}\right) \quad \text { in } \quad \mathbb{R}^{N} \\
v_{n} \in H^{1}\left(\mathbb{R}^{N}\right) .
\end{array}\right.
$$

We consider $\eta \in C_{c}^{\infty}\left(\mathbb{R}^{N}\right), L>0$ and $\beta>1$ arbitrary, and define $z_{L, n}:=\eta^{2} v_{L, n}^{2(\beta-1)} v_{n,+}$ and $w_{L, n}:=\eta v_{n,+} v_{L, n}^{\beta-1}$ where $v_{L, n}=\min \left\{v_{n,+}, L\right\}$. Applying $z_{L, n}$ as a test function, we find

$$
\begin{aligned}
& \int \eta^{2} v_{L, n}^{2(\beta-1)}\left|\nabla v_{n,+}\right|^{2} d x \leq|A|_{\infty} \int\left|f\left(v_{n}\right)\right| \eta^{2} v_{L, n}^{2(\beta-1)} v_{n,+} d x- \\
& -\int V(x) v_{n} v_{L, n}^{2(\beta-1)} \eta^{2} v_{n,+} d x-2 \int\left(\nabla v_{n} \nabla \eta\right) \eta v_{L, n}^{2(\beta-1)} v_{n,+} d x
\end{aligned}
$$

Since

$$
\begin{gathered}
\left|\int v_{L, n}^{2(\beta-1)}\left(v_{n,+} \nabla \eta\right)\left(\eta \nabla v_{n}\right) d x\right| \leq C \int v_{L, n}^{2(\beta-1)} v_{n,+}^{2}|\nabla \eta|^{2} d x+ \\
+\frac{1}{4} \int v_{L, n}^{2(\beta-1)} \eta^{2}\left|\nabla v_{n,+}\right|^{2} d x
\end{gathered}
$$

combining (2.24), (2.25) and (2.26), we obtain

$$
\begin{aligned}
& \int \eta^{2} v_{L, n}^{2(\beta-1)}\left|\nabla v_{n,+}\right|^{2} d x \leq C \int\left|v_{n,+}\right|^{2} \eta^{2} v_{L, n}^{2(\beta-1)} d x+ \\
& +C \int\left|v_{n}\right|^{2^{*}} \eta^{2} v_{L, n}^{2(\beta-1)} d x+C \int v_{L, n}^{2(\beta-1)} v_{n,+}^{2}|\nabla \eta|^{2} d x
\end{aligned}
$$

where $C>0$ is independently of $\beta>1, \eta \in C_{c}^{\infty}\left(\mathbb{R}^{N}\right)$ and $L>0$.

On the other hand, since $H^{1}\left(\mathbb{R}^{N}\right) \hookrightarrow D^{1,2}\left(\mathbb{R}^{N}\right) \hookrightarrow L^{2^{*}}\left(\mathbb{R}^{N}\right)$,

$$
\begin{gathered}
\left|w_{L, n}\right|_{2^{*}}^{2} \leq C \int\left|\nabla w_{L, n}\right|^{2} d x \leq C \int|\nabla \eta|^{2} v_{L, n}^{2(\beta-1)} v_{n,+}^{2} d x+ \\
C \int \eta^{2} v_{L, n}^{2(\beta-1)}\left|\nabla v_{n,+}\right|^{2} d x+C \int \eta^{2}\left|\nabla v_{L, n}^{(\beta-1)}\right|^{2} v_{n,+}^{2} d x,
\end{gathered}
$$

and thus

$$
\left|w_{L, n}\right|_{2^{*}}^{2} \leq C \beta^{2}\left(\int|\nabla \eta|^{2} v_{L, n}^{2(\beta-1)} v_{n,+}^{2} d x+\int \eta^{2} v_{L, n}^{2(\beta-1)}\left|\nabla v_{n,+}\right|^{2} d x\right) .
$$

Then, from (2.27) and (2.29),

$$
\left|w_{L, n}\right|_{2^{*}}^{2} \leq C \beta^{2}\left(\int\left|v_{n,+}\right|^{2} \eta^{2} v_{L, n}^{2(\beta-1)} d x+\right.
$$




$$
\left.+\int\left|v_{n}\right|^{2^{*}} \eta^{2} v_{L, n}^{2(\beta-1)} d x+\int v_{L, n}^{2(\beta-1)} v_{n,+}^{2}|\nabla \eta|^{2} d x\right),
$$

where $C>0$ is independently of $n \in \mathbb{N}, \beta>1, L>0$ and $\eta \in C_{c}^{\infty}\left(\mathbb{R}^{N}\right)$.

Claim 2.14 There exists $R>0$ such that

$$
\sup _{n \in \mathbb{N}, x \in \mathbb{R}^{N}} \int_{B_{3 R}(x)} v_{n,+}^{\frac{2^{* 2}}{2}} d x<+\infty .
$$

In fact, fix $\beta_{0}:=\frac{2^{*}}{2}$. By using the limit $v_{n} \rightarrow v$ in $L^{2^{*}}\left(\mathbb{R}^{N}\right)$, we can fix $R>0$ sufficiently small verifying

$$
C \beta_{0}^{2}\left(\int_{B_{4 R}(x)} v_{n,+}^{2^{*}} d x\right)^{\frac{2^{*}-2}{2}}<\frac{1}{2}, \text { for all } n \in \mathbb{N} \text { and } x \in \mathbb{R}^{N},
$$

where $C$ is given in (2.30). On the other hand, consider $\eta_{x} \in C_{c}^{\infty}\left(\mathbb{R}^{N},[0,1]\right)$ such that $\eta_{x} \equiv 1$ on $B_{3 R}(x), \eta_{x} \equiv 0$ on $\mathbb{R}^{N} \backslash B_{4 R}(x)$ and $x \mapsto\left\|\nabla \eta_{x}\right\|_{\infty}$ is a constant function. Then,

$$
\begin{aligned}
& \int v_{n,+}^{2^{*}} \eta_{x}^{2} v_{L, n}^{2\left(\beta_{0}-1\right)}=\int v_{n,+}^{2^{*}} \eta_{x}^{2} v_{L, n}^{2^{*}-2}=\int_{B_{4 R}(x)}\left(v_{n,+}^{2} \eta_{x}^{2} v_{L, n}^{2^{*}-2}\right) v_{n,+}^{2^{*}-2} d x \leq \\
& \leq\left(\int\left(v_{n,+} \eta_{x} v_{L, n}^{\frac{2^{*}-2}{2}}\right)^{2^{*}} d x\right)^{\frac{2}{2^{*}}}\left(\int_{B_{4 R}(x)} v_{n,+}^{2^{*}} d x\right)^{\frac{2^{*}-2}{2}} \leq \frac{1}{2 C \beta_{0}^{2}}\left|w_{L, n}\right|_{2^{*}}^{2}
\end{aligned}
$$

Applying (2.30) with $\eta=\eta_{x}$ and $\beta=\beta_{0}$, we get

$$
\left|w_{L, n}\right|_{2^{*}}^{2} \leq C \beta_{0}^{2}\left(\int \eta_{x}^{2} v_{n,+}^{2^{*}} d x+\frac{1}{2 C \beta_{0}}\left|w_{L, n}\right|_{2^{*}}^{2}+\int v_{n,+}^{2^{*}}\left|\nabla \eta_{x}\right|^{2} d x\right),
$$

which leads to

$$
\left|w_{L, n}\right|_{2^{*}}^{2} \leq C \beta_{0}^{2}\left(1+\left\|\nabla \eta_{x}\right\|_{\infty}\right) \int v_{n,+}^{2^{*}} d x
$$

By using Fatou's Lemma for $L \rightarrow+\infty$, we obtain

$$
\left(\int_{B_{3 R}(x)} v_{n,+}^{\frac{2^{* 2}}{2}} d x\right)^{\frac{2}{2^{*}}} \leq C \beta_{0}^{2} \int v_{n,+}^{2^{*}} d x
$$

for all $n \in \mathbb{N}$ and for all $x \in \mathbb{R}^{N}$. This proves Claim 2.14

In what follows, we fix $R>0$ as in Claim 2.14, $r_{m}:=\frac{2 R}{2^{m}}$,

$$
t:=\frac{2^{* 2}}{2\left(2^{*}-2\right)} \quad \text { and } \quad \chi:=\frac{2^{*}(t-1)}{2 t}>1 .
$$


Claim 2.15 Consider $\beta>1$ arbitrary such that $v_{n,+} \in L^{\beta \frac{2^{*}}{\chi}}\left(B_{R+r_{m}}(x)\right)$ for all $n \in \mathbb{N}$ and for some $m \in \mathbb{N}$. Then

$$
\left|v_{n,+}\right|_{L^{2^{*} \beta}\left(B_{R+r_{m+1}}(x)\right)} \leq C^{1 / \beta} \beta^{1 / 2 \beta}\left(1+4^{m}\right)^{1 / 2 \beta}\left|v_{n,+}\right|_{L^{2^{*} \frac{\beta}{\chi}}\left(B_{R+r_{m}}(x)\right)}
$$

where $C>0$ is independently of $n, m \in \mathbb{N}, \beta>1$ and $x \in \mathbb{R}^{N}$.

In fact, since $2^{*} \frac{\beta}{\chi}=\beta \frac{2 t}{t-1}, v_{n,+} \in L^{\frac{2 \beta t}{t-1}}\left(B_{R+r_{m}}(x)\right)$ for all $n \in \mathbb{N}$. Consider $\eta_{x, m} \in C_{c}^{\infty}\left(\mathbb{R}^{N},[0,1]\right)$ such that $\eta_{x, m} \equiv 1$ in $B_{R+r_{m+1}}(x), \eta_{x, m} \equiv 0$ in $\mathbb{R}^{N} \backslash B_{R+r_{m}}(x)$ and $\left|\eta_{x, m}\right|_{\infty}<\frac{2}{r_{m+1}}$. Using $\eta=\eta_{x, m}$ in (2.30),

$$
\begin{gathered}
\left|w_{L, n}\right|_{2^{*}}^{2} \leq C \beta^{2}\left(\int_{B_{R+r_{m}}(x)}\left|v_{n,+}\right|^{2 \beta} d x+\int_{B_{R+r_{m}}(x)} v_{n,+}^{2^{*}-2} v_{n,+}^{2 \beta} d x+\right. \\
\left.+\left(\frac{2}{r_{m+1}}\right)^{2} \int_{B_{R+r_{m}}(x)} v_{n,+}^{2 \beta} d x\right) \leq C \beta^{2}\left(\left(1+4^{m}\right) \int_{B_{R+r_{m}}(x)} v_{n,+}^{2 \beta} d x+\right. \\
\left.+\int_{B_{R+r_{m}}(x)} v_{n,+}^{2^{*}-2} v_{n,+}^{2 \beta} d x\right) \leq C \beta^{2}\left(\left(1+4^{m}\right)\left(\int_{B_{3 R}(0)} 1 d x\right)^{1 / t} \cdot\right. \\
\cdot\left(\int_{B_{R+r_{m}}(x)} v_{n,+}^{2 \beta t /(t-1)} d x\right)^{(t-1) / t}+\left(\int_{B_{3 R}(x)} v_{n,+}^{\left(2^{*}-2\right) t} d x\right)^{1 / t} \cdot \\
\left.\left(\int_{B_{R+r_{m}}(x)} v_{n,+}^{2 \beta t /(t-1)} d x\right)^{(t-1) / t}\right)^{\leq C \beta^{2}}\left(\left(1+4^{m}\right)\left(\int_{B_{R+r_{m}}(x)} v_{n,+}^{2 \beta t /(t-1)} d x\right)^{(t-1) / t}\right)^{.}
\end{gathered}
$$

Thus

$$
\left|w_{L, n}\right|_{2^{*}}^{2} \leq C \beta^{2}\left(1+4^{m}\right)\left|v_{n,+}\right|_{L^{2 \beta t /(t-1)}\left(B_{R+r_{m}}(x)\right)}^{2 \beta} .
$$

Applying Fatou's Lemma as $L \rightarrow+\infty$ we get (2.32). Consequently, by induction,

$$
\left|v_{n,+}\right|_{L^{2^{*} \chi^{m}}\left(B_{R+r_{m+1}}(x)\right)} \leq C^{\sum_{i=1}^{m} \frac{1}{\chi^{i}}} \chi^{\sum_{i=1}^{m} \frac{i}{2 \chi^{i}}} \prod_{i=1}^{m}\left(1+4^{i}\right)^{\frac{1}{2 \chi^{i}}}\left|v_{n,+}\right|_{L^{2^{*}}\left(B_{2 R}(x)\right)}
$$


Since $\left(\sum_{i=1}^{m} \frac{1}{\chi^{i}}\right)_{m}$ and $\left(\sum_{i=1}^{m} \frac{i}{\chi^{i}}\right)_{m}$ are convergent because $\chi>1$, and that

$$
\prod_{i=1}^{m}\left(1+4^{i}\right)^{\frac{1}{2 \chi^{i}}}=4^{\sum_{i=1}^{m} \frac{\log _{4}\left(1+4^{i}\right)}{2 \chi^{i}}} \leq 4^{\sum_{i=1}^{m} \frac{\log _{4}\left(4^{i+1}\right)}{2 \chi^{i}}}=4^{\sum_{i=1}^{m} \frac{i+1}{2 \chi^{i}}},
$$

there exists $C>0$ independently of $n, m \in \mathbb{N}$ and $x \in \mathbb{R}^{N}$ such that

$$
\left|v_{n,+}\right|_{L^{2^{*}} \chi^{m}\left(B_{R}(x)\right)} \leq C\left|v_{n,+}\right|_{L^{2^{*}}\left(B_{2 R}(x)\right)} .
$$

Now (2.22) follows by taking the limit of $m \rightarrow+\infty$.

Corollary 2.16 For each $\delta>0$ there exist $R>0$ such that $\left|v_{n}(x)\right| \leq \delta$ for all $x \in \mathbb{R}^{N} \backslash B_{R}(0)$ and $n \in \mathbb{N}$.

Proof. By Lemma 2.13.

$$
\left|v_{n}\right|_{L^{\infty}\left(B_{R}(x)\right)} \leq C\left|v_{n}\right|_{L^{2^{*}}\left(B_{2 R}(x)\right)}, \quad \text { for all } \quad n \in \mathbb{N} \quad \text { and } \quad x \in \mathbb{R}^{N} .
$$

This fact combined with the limit $v_{n} \rightarrow v$ in $L^{2^{*}}\left(\mathbb{R}^{N}\right)$ proves the result.

\section{Concentration of the solutions:}

As $v \neq 0$, we must have $\left|v_{n}\right|_{L^{\infty}\left(\mathbb{R}^{N}\right)} \not \nrightarrow 0$. Hence, we can assume that $\left|v_{n}\right|_{L^{\infty}\left(\mathbb{R}^{N}\right)}>\delta$ for any $\delta>0$ and $n \in \mathbb{N}$. In what follows, we fix $z_{n} \in \mathbb{R}^{N}$ verifying

$$
\left|v_{n}\left(z_{n}\right)\right|=\max _{x \in \mathbb{R}^{N}}\left|v_{n}(x)\right| .
$$

Since $v_{n}(x)=u_{n}\left(x+y_{n}\right)$, the point $x_{n}:=z_{n}+y_{n}$ satisfies

$$
\left|u_{n}\left(x_{n}\right)\right|=\max _{x \in \mathbb{R}^{N}}\left|u_{n}(x)\right| .
$$

From Corollary 2.16, $\left(z_{n}\right)$ is bounded in $\mathbb{R}^{N}$, then

$$
\epsilon_{n} x_{n}=\epsilon_{n} z_{n}+\epsilon_{n} y_{n} \rightarrow z \in \mathcal{A}
$$

and

$$
\lim _{n} A\left(\epsilon_{n} x_{n}\right)=A(z)=A(0)
$$




\section{The case $N=2$.}

In this section we will consider the case where $f$ has an exponential critical growth. For this type of function, it is well known that Trundiger-Moser type inequalities are key points to apply variational methods. In the present paper we will use a Trudinger-Moser type inequality for whole $\mathbb{R}^{2}$ due to Cao 4] ( see also Ruf [21]).

Lemma 3.1 (Trudinger-Moser inequality for unbounded domains) For all $u \in H^{1}\left(\mathbb{R}^{2}\right)$, we have

$$
\int\left(e^{\alpha|u|^{2}}-1\right) d x<\infty, \quad \text { for every } \alpha>0
$$

Moreover, if $|\nabla u|_{2}^{2} \leq 1,|u|_{2} \leq M<\infty$ and $\alpha<4 \pi$, then there exists a positive constant $C=C(M, \alpha)$ such that

$$
\int\left(e^{\alpha|u|^{2}}-1\right) d x \leq C
$$

The reader can find other Trundiger-Moser type inequalities in [6], 11], [12], [18] and references therein

As in the previous section, firstly we need to study the autonomous case.

\subsection{A result involving the autonomous problem.}

We consider the problem

$$
\left\{\begin{array}{l}
-\Delta u+V(x) u=\lambda f(u), \quad x \in \mathbb{R}^{2}, \quad(A P)_{\lambda}^{\exp } \\
u \in H^{1}\left(\mathbb{R}^{2}\right),
\end{array}\right.
$$

where $f: \mathbb{R} \rightarrow \mathbb{R}$ satisfies $\left(f_{1}\right)-\left(f_{5}\right)$. Associated with this problem, we have the energy function $J_{\lambda}: H^{1}\left(\mathbb{R}^{2}\right) \rightarrow \mathbb{R}$ given by

$$
J_{\lambda}(u)=\frac{1}{2}\left\|u^{+}\right\|^{2}-\frac{1}{2}\left\|u^{-}\right\|^{2}-\lambda \int F(u) d x .
$$

It is well known that $J_{\lambda} \in C^{1}\left(H^{1}\left(\mathbb{R}^{2}\right), \mathbb{R}\right)$ with

$$
J_{\lambda}^{\prime}(u) v=B(u, v)-\lambda \int f(u) v d x, \quad \forall u, v \in H^{1}\left(\mathbb{R}^{2}\right) .
$$


In the sequel,

$$
\mathcal{N}_{\lambda}=\left\{u \in H^{1}\left(\mathbb{R}^{2}\right) \backslash E^{-} ; J_{\lambda}^{\prime}(u) u=J_{\lambda}^{\prime}(u) v=0, \forall v \in E^{-}\right\}
$$

and

$$
d_{\lambda}=\inf _{\mathcal{N}_{\lambda}} J_{\lambda}
$$

In [1], Alves and Germano have proved that there exists a constant $\tau_{0}>0$ such that $(A P)_{\lambda}^{e x p}$ has a ground state solution if

$$
\lambda \geq A(0) \text { and } \tau \geq \tau_{0},
$$

where $\tau$ was fixed in $\left(f_{5}\right)$. More precisely, it has been shown that for $\lambda \geq A(0)$ and $\tau \geq \tau_{0}$, there exists $u_{\lambda} \in H^{1}\left(\mathbb{R}^{2}\right)$ verifying

$$
J_{\lambda}^{\prime}\left(u_{\lambda}\right)=0 \text { and } J_{\lambda}\left(u_{\lambda}\right)=d_{\lambda}
$$

with

$$
d_{\lambda}<\frac{\widetilde{A}^{2}}{2}
$$

where $\widetilde{A}<1 / a$ and $a$ was given in (1.3). This restriction on $\tau$ has been mentioned in Theorem 1.1, and it will be assume in whole this section.

Moreover, the authors have proved that for all $u \in H^{1}\left(\mathbb{R}^{2}\right) \backslash E^{-}$the set $\mathcal{N}_{\lambda} \cap \widehat{E}(u)$ is a singleton set and the element of this set is the unique global maximum of $\left.J_{\lambda}\right|_{\widehat{E}(u)}$, which means precisely that there exist uniquely $t^{*} \geq 0$ and $v^{*} \in E^{-}$such that

$$
J_{\lambda}\left(t^{*} u+v^{*}\right)=\max _{w \in \widehat{E}(u)} J_{\lambda}(w) \quad \text { and } \quad\left\{t^{*} u+v^{*}\right\}=\mathcal{N}_{\lambda} \cap \widehat{E}(u)
$$

As in the case $N \geq 3$, we begin by studying the behavior of the function $\lambda \mapsto d_{\lambda}$.

Proposition 3.2 The function $\lambda \mapsto d_{\lambda}$ is decreasing and continuous on $\left[A_{0},+\infty\right)$.

Proof. The monotonicity of $\lambda \mapsto d_{\lambda}$ and some details of the proof are analogous to Proposition 2.1 and [2, Proposition 2.3]. In order to get the $\operatorname{limit} \lim _{n} d_{\lambda_{n}}=d_{\lambda}$, it suffices to consider $\lambda_{1} \geq \lambda_{2} \geq \ldots \geq \lambda_{n} \rightarrow \lambda$. Let $u_{n}$ be a ground state solution of the problem $(A P)_{\lambda_{n}}^{\exp }$. Let $t_{n} \geq 0$ and $v_{n} \in E^{-}$ such that $t_{n} u_{n}+v_{n} \in \mathcal{N}_{\lambda}$. Consequently

$$
J_{\lambda}\left(t_{n} u_{n}+v_{n}\right)=\max _{\widehat{E}\left(u_{n}\right)} J_{\lambda} \geq d_{\lambda}
$$


and the same ideas explored in Proposition 2.1 remain valid to show that $\left(\int f\left(u_{n}\right) u_{n} d x\right)$ is bounded in $\mathbb{R}$. Now, arguing as in [1, Lemma 3.11], we see that $\left(u_{n}\right)$ is bounded in $H^{1}\left(\mathbb{R}^{2}\right)$.

Note that there exist $\left(y_{n}\right)$ in $\mathbb{Z}^{2}, r, \eta>0$ such that

$$
\int_{B_{r}\left(y_{n}\right)}\left|u_{n}^{+}\right|^{2} d x>\eta, \quad \forall n \in \mathbb{N} .
$$

Otherwise, $u_{n}^{+} \rightarrow 0$ in $L^{p}\left(\mathbb{R}^{2}\right)$ for all $p>2$. Defining $w_{n}(x):=\widetilde{A} \frac{u_{n}^{+}(x)}{\left\|u_{n}\right\|}$ where $\widetilde{A}$ was given in (3.35), we have

$$
\left\|w_{n}\right\|_{H^{1}\left(\mathbb{R}^{2}\right)} \leq \widetilde{A} a<1, \quad \forall n \in \mathbb{N} .
$$

This fact permits to repeat the same approach found in [3, Proposition 2.3] to get the limit

$$
\int F\left(w_{n}\right) d x \rightarrow 0
$$

As $w_{n} \in \widehat{E}\left(u_{n}\right)$ and $u_{n} \in \mathcal{N}_{\lambda_{n}}$, it follows that

$$
d_{\lambda} \geq d_{\lambda_{n}}=J_{\lambda_{n}}\left(u_{n}\right) \geq J_{\lambda_{n}}\left(w_{n}\right)=\frac{\widetilde{A}}{2}-\lambda_{n} \int F\left(w_{n}\right) d x .
$$

Passing to the limit as $n \rightarrow+\infty$ we obtain $d_{\lambda} \geq \widetilde{A} / 2$, which contradicts (3.35), and (3.36) holds. If $\widetilde{u}_{n}(x):=u_{n}\left(x+y_{n}\right)$, then $\widetilde{u}_{n}^{+}(x):=u_{n}^{+}\left(x+y_{n}\right)$, and by (3.36), $\widetilde{u}_{n}^{+} \rightarrow u \neq 0$. This implies that $\mathcal{V}:=\left\{\widetilde{u}_{n}^{+}\right\}_{n \in \mathbb{N}}$ satisfies $0 \notin \overline{\mathcal{V}}^{\sigma\left(H^{1}\left(\mathbb{R}^{2}\right), H^{1}\left(\mathbb{R}^{2}\right)^{\prime}\right)}$ and $\mathcal{V}$ is bounded in $H^{1}\left(\mathbb{R}^{2}\right)$. We proceed as in Proposition 2.1 to conclude $\left(t_{n} u_{n}+v_{n}\right)$ is bounded and $d_{\lambda_{n}} \leq d_{\lambda}+o_{n}$. This finishes the proof.

\subsection{Existence of ground state for problem $(P)_{\epsilon}$.}

The three first results this section follow as in the case $N \geq 3$, then we will omit their proofs.

Lemma 3.3 The limit $\lim _{\epsilon \rightarrow 0} c_{\epsilon}=c_{0}$ holds. Moreover, if $w_{0}$ is a ground state solution of the problem $(P)_{0}$ and let $t_{\epsilon} \geq 0$ and $v_{\epsilon} \in E^{-}$such that $t_{\epsilon} w_{0}+v_{\epsilon} \in \mathcal{M}_{\epsilon}$. Then

$$
t_{\epsilon} \rightarrow 1 \text { and } v_{\epsilon} \rightarrow 0
$$

as $\epsilon \rightarrow 0$. 
Corollary 3.4 There exists $\epsilon_{0}>0$ such that

$$
c_{\epsilon}<d_{A_{\infty}} \text { and } c_{\epsilon}<\frac{\widetilde{A}^{2}}{2}, \text { for all } \epsilon \in\left(0, \epsilon_{0}\right) .
$$

Proposition 3.5 There exists a bounded sequence $\left(u_{n}\right) \subset \mathcal{M}_{\epsilon}$ such that $\left(u_{n}\right)$ is $(P S)_{c_{\epsilon}}$ for $I_{\epsilon}$.

Now we are ready to prove the existence of solution for $\epsilon$ small enough.

Theorem 3.6 Problem $(P)_{\epsilon}$ has a ground state solution for $\epsilon \in\left(0, \epsilon_{0}\right)$.

Proof. To begin with, we claim that there are $\left(z_{n}\right) \subset \mathbb{Z}^{2}$ and $r, \eta>0$ such that

$$
\int_{B_{r}\left(z_{n}\right)}\left|u_{n}^{+}\right|^{2} d x>\eta, \quad \forall n \in \mathbb{N} .
$$

In fact, if the claim does not hold, we must have $u_{n}^{+} \rightarrow 0$ in $L^{p}\left(\mathbb{R}^{2}\right)$ for all $p \in(2,+\infty)$. Since $u_{n} \in \mathcal{M}_{\epsilon}$, by (2.16), $\left\|u_{n}^{+}\right\|^{2} \geq 2 c_{\epsilon} \geq 2 c_{0}$. Setting $\widetilde{w}_{n}(x):=\widetilde{A} \frac{u_{n}^{+}}{\left\|u_{n}^{+}\right\|}$and arguing as in Proposition 3.2 , we find $c_{\epsilon} \geq \frac{\widetilde{A}^{2}}{2}$, which is a contradiction. Therefore (3.37) holds.

Claim $3.7\left(z_{n}\right)$ is bounded in $\mathbb{R}^{2}$.

Suppose $\left|z_{n}\right| \rightarrow+\infty$ and define $w_{n}(x):=u_{n}\left(x+z_{n}\right)$. From (3.37), we can suppose that $w_{n} \rightarrow w \neq 0$ in $H^{1}\left(\mathbb{R}^{2}\right)$. As it was done in (2.10), $\left(\int f\left(w_{n}\right) w_{n} d x\right)$ is bounded in $L^{1}\left(\mathbb{R}^{2}\right)$. By [7, Lemma 2.1],

$$
f\left(w_{n}\right) \rightarrow f(w) \text { in } L^{1}(B),
$$

for all $B \subset \mathbb{R}^{2}$ bounded Borel set. Now, we repeat the same idea explored in Claim 2.7 to deduce that $w$ is a critical point of $J_{A_{\infty}}$ with $d_{A_{\infty}} \leq c_{\epsilon}$, which is absurd. This proves the Claim 3.7 .

To conclude the proof we proceed as in Theorem 2.6 to prove that the weak limit of $\left(u_{n}\right)$ is a ground state solution for $I_{\epsilon}$.

\subsection{Concentration of the solutions.}

In this section we fix $\epsilon_{n} \rightarrow 0$ with $\epsilon_{n} \in\left(0, \epsilon_{0}\right)$ for all $n \in \mathbb{N}$. By results of the last section, for each $n \in \mathbb{N}$ there exists $u_{n}$ in $H^{1}\left(\mathbb{R}^{2}\right)$ such that

$$
I_{n}\left(u_{n}\right)=c_{n} \text { and } I_{n}^{\prime}\left(u_{n}\right)=0,
$$

with the notation $I_{n}:=I_{\epsilon_{n}}$ and $c_{n}:=c_{\epsilon_{n}}$. 
Lemma 3.8 The sequence $\left(u_{n}\right)$ is bounded in $H^{1}\left(\mathbb{R}^{2}\right)$.

Proof. See proof of [1, Lemma 3.11].

Lemma 3.9 There are $r, \eta>0$ and $\left(y_{n}\right) \subset \mathbb{Z}^{2}$ such that

$$
\int_{B_{r}\left(y_{n}\right)}\left|u_{n}^{+}\right|^{2} d x>\eta
$$

Proof. See proof of (3.37).

From now on, we set $v_{n}(x):=u_{n}\left(x+y_{n}\right)$. Then, by (3.38), $v_{n} \rightarrow v \neq 0$ in $H^{1}\left(\mathbb{R}^{2}\right)$ for some subsequence.

Lemma 3.10 The sequence $\left(\epsilon_{n} y_{n}\right)$ is bounded in $\mathbb{R}^{2}$. Moreover, $I_{0}^{\prime}(v)=0$ and if $\epsilon_{n} y_{n} \rightarrow z \in \mathbb{R}^{2}$ then $z \in \mathcal{A}$ or equivalently $A(z)=A(0)$.

Proof. As in the previous section, $\left(f\left(u_{n}\right) u_{n}\right)$ is bounded in $L^{1}\left(\mathbb{R}^{2}\right)$. Then, by [7, Lemma 2.1],

$$
f\left(u_{n}\right) \rightarrow f(u) \text { in } L^{1}(B),
$$

for all bounded Borel set $B \subset \mathbb{R}^{2}$. The above limit permits to repeat the same arguments explored in Lemma 2.10.

Our next proposition follows with the same idea explored in Proposition 2.11, then we omit its proof.

Proposition 3.11 There exists $h \in L^{1}\left(\mathbb{R}^{2}\right)$ and a subsequence of $\left(v_{n}\right)$ such that

$$
\left|f\left(v_{n}(x)\right) v_{n}(x)\right| \leq h(x), \quad \text { for all } \quad x \in \mathbb{R}^{2} \quad \text { and } n \in \mathbb{N} .
$$

As an immediate consequence of the last lemma, we have the following corollary

Corollary $3.12 v_{n} \rightarrow v$ in $L^{q}\left(\mathbb{R}^{2}\right)$ where $q$ was given in $\left(f_{5}\right)$.

Proof. It suffices to note that $f\left(v_{n}\right) v_{n} \geq \theta F\left(v_{n}\right) \geq \theta \tau\left|v_{n}\right|^{q}$ for all $n \in \mathbb{N}$ and $v_{n}(x) \rightarrow v(x)$ a.e in $\mathbb{R}^{N}$.

The next lemma have been motivated by an inequality found $\underline{8}$, Lemma 2.11], however it is a little different, because we need to adapt it for our problem. 
Lemma 3.13 For all $t, s \geq 0$ and $\beta \in(0,1]$,

$$
t s \leq \begin{cases}4\left(e^{t^{2}}-1\right)\left(\ln ^{+} s\right)+s\left(\ln ^{+} s\right)^{1 / 2}, & \text { if } s>e^{1 / 4} \\ e^{1 / 4} t s^{\beta}, & \text { if } s \in\left[0, e^{1 / 4}\right] .\end{cases}
$$

Proof. From [8, Lemma 2.11], if $s>e^{1 / 4}$ then $l n^{+} s>1 / 4$ and

$$
t s \leq\left(e^{t^{2}}-1\right)+s\left(\ln ^{+} s\right)^{1 / 2} \leq 4\left(e^{t^{2}}-1\right)\left(\ln ^{+} s\right)+s\left(\ln ^{+} s\right)^{1 / 2} .
$$

For $s \in[0,1)$, we have $t s \leq t s^{\beta} \leq e^{1 / 4} t s^{\beta}$, and if $s \in\left[1, e^{1 / 4}\right]$, then $t s \leq t e^{1 / 4} \leq e^{1 / 4} t s^{\beta}$. This proves the inequality.

Proposition $3.14 v_{n} \rightarrow v$ in $H^{1}\left(\mathbb{R}^{2}\right)$.

Proof. To begin with, by $\left(f_{1}\right)$, there exists $K>0$ such that

$$
|f(t)| \leq \Gamma e^{1 / 4} \Longrightarrow|f(t)|^{2} \leq K f(t) t .
$$

On the other hand,

$$
\begin{gathered}
\left(\left|f\left(v_{n}\right)\right| \chi_{\left[0, e^{1 / 4}\right]}\left(\frac{1}{\Gamma}\left|f\left(v_{n}\right)\right|\right)\right)^{2}=\left|f\left(v_{n}\right)\right|^{2} \chi_{\left[0, \Gamma e^{1 / 4}\right]}\left(\left|f\left(v_{n}\right)\right|\right) \leq \\
\leq K f\left(v_{n}\right) v_{n} \leq K h \in L^{1}\left(\mathbb{R}^{2}\right) .
\end{gathered}
$$

Thus, there exists $\widetilde{h} \in L^{2}\left(\mathbb{R}^{2}\right)$ such that

$$
\left|f\left(v_{n}\right)\right| \chi_{\left[0, e^{1 / 4}\right]}\left(\frac{1}{\Gamma}\left|f\left(v_{n}\right)\right|\right) \leq \widetilde{h}, \quad \forall n \in \mathbb{N} .
$$

In what follows, fixing $\alpha>0$ such that $\frac{\alpha^{2} q}{q-1} \sup _{n \in \mathbb{N}}\left\|v_{n}^{+}\right\|_{H^{1}\left(\mathbb{R}^{2}\right)}^{2}<1$, the Lemma 3.1 guarantees that

$$
b_{n}:=\left(e^{\alpha^{2}\left|v_{n}^{+}\right|^{2}}-1\right) \in L^{\frac{q}{q-1}}\left(\mathbb{R}^{2}\right) \quad \text { and } \quad\left|b_{n}\right|_{\frac{q}{q-1}} \leq C
$$

for all $n \in \mathbb{N}$ and some $C>0$. Applying the Lemma 3.13 for $t=\alpha\left|v_{n}^{+}\right|$, $s=\frac{1}{\Gamma}\left|f\left(v_{n}\right)\right|$ and $\beta=1$, we obtain

$$
\left|f\left(v_{n}\right) v_{n}^{+}\right|=\frac{\Gamma}{\alpha} \frac{\left|f\left(v_{n}\right)\right|}{\Gamma} \alpha\left|v_{n}^{+}\right| \leq \frac{\Gamma}{\alpha} 4\left(e^{\alpha^{2}\left|v_{n}^{+}\right|^{2}}-1\right)\left(\ln +\left(\frac{1}{\Gamma}\left|f\left(v_{n}\right)\right|\right)\right)+
$$




$$
\begin{gathered}
+\frac{1}{\alpha}\left|f\left(v_{n}\right)\right|\left(\ln +\left(\frac{1}{\Gamma}\left|f\left(v_{n}\right)\right|\right)\right)^{1 / 2}+e^{1 / 4}\left|v_{n}^{+}\right|\left|f\left(v_{n}\right)\right| \chi_{\left[0, e^{1 / 4}\right]}\left(\frac{1}{\Gamma} f\left(v_{n}\right)\right) \leq \\
\leq \frac{16 \Gamma \pi}{\alpha} b_{n}\left|v_{n}\right|^{2}+\frac{\sqrt{4 \pi}}{\alpha} f\left(v_{n}\right) v_{n}+e^{1 / 4}\left|v_{n}^{+}\right| \widetilde{h} .
\end{gathered}
$$

Since $b_{n} \rightarrow b$ in $L^{\frac{q}{q-1}}\left(\mathbb{R}^{2}\right)$ and $v_{n} \rightarrow v$ in $L^{q}\left(\mathbb{R}^{2}\right)$, we have that $\left(b_{n}\left|v_{n}\right|^{2}\right)$ is strongly convergent in $L^{1}\left(\mathbb{R}^{2}\right)$. Here, we have used the fact that $b_{n}\left|v_{n}\right|^{2} \geq 0$ and $v_{n}(x) \rightarrow v(x)$ a.e in $\mathbb{R}^{N}$. Analogously $\left(\left|v_{n}^{+}\right| \widetilde{h}\right)$ converges in $L^{1}\left(\mathbb{R}^{2}\right)$. Consequently there is $H_{1} \in L^{1}\left(\mathbb{R}^{2}\right)$ such that, for some subsequence,

$$
\left|f\left(v_{n}\right) v_{n}^{+}\right| \leq H, \quad \forall n \in \mathbb{N} .
$$

The same argument works to show that there exists $H_{2} \in L^{1}\left(\mathbb{R}^{2}\right)$ such that, for some subsequence,

$$
\left|f\left(v_{n}\right) v_{n}^{-}\right| \leq H_{2}, \quad \forall n \in \mathbb{N} .
$$

As an consequence of the above information,

$$
f\left(v_{n}\right) v_{n}^{+} \rightarrow f(v) v^{+} \quad \text { and } \quad f\left(v_{n}\right) v_{n}^{-} \rightarrow f\left(v_{n}\right) v^{-} \text {in } L^{1}\left(\mathbb{R}^{2}\right) .
$$

Now, recalling that $I_{0}^{\prime}(v)=I_{n}^{\prime}\left(v_{n}\right) v_{n}^{+}=I_{n}^{\prime}\left(v_{n}\right) v_{n}^{-}=0, v_{n}^{+} \rightarrow v^{+}$, and $v_{n}^{-} \rightarrow v^{-}$in $H^{1}\left(\mathbb{R}^{2}\right)$, we get the desired result.

Lemma 3.15 For all $n \in \mathbb{N}, v_{n} \in C\left(\mathbb{R}^{2}\right)$. Moreover, there exist $G \in$ $L^{3}\left(\mathbb{R}^{2}\right), C>0$ independently of $x \in \mathbb{R}^{2}$ and $n \in \mathbb{N}$ such that

$$
\left\|v_{n}\right\|_{C\left(\overline{B_{1}(x)}\right)} \leq C|G|_{L^{3}\left(B_{2}(x)\right)}, \quad \text { for all } \quad n \in \mathbb{N} \quad \text { and } \quad x \in \mathbb{R}^{2} .
$$

Hence, there exists $C>0$ such that $\left|v_{n}\right|_{L^{\infty}\left(\mathbb{R}^{2}\right)} \leq C$ and

$$
\left|v_{n}(x)\right| \rightarrow 0 \text { as }|x| \rightarrow+\infty \text {, uniformly in } n \in \mathbb{N} .
$$

Proof. We know that there are $C_{1}, C_{2}>0$ such that

$$
|f(t)| \leq C_{1}|t|+C_{2}\left(e^{5 \pi t^{2}}-1\right) \quad \forall t \in \mathbb{R} .
$$

By Proposition 3.14, there exists $H \in H^{1}\left(\mathbb{R}^{2}\right)$ such that $\left|v_{n}(x)\right| \leq H(x)$ for all $n \in \mathbb{N}$ and $x \in \mathbb{R}^{2}$. Setting

$$
G:=\left(\|V\|_{\infty}+A(0) C_{1}\right) H+A(0) C_{2}\left(e^{5 \pi H^{2}}-1\right) \in L^{3}\left(\mathbb{R}^{2}\right)
$$

it follows that

$$
\left|A\left(\epsilon_{n} x+\epsilon_{n} y_{n}\right) f\left(v_{n}\right)-V(x) v_{n}\right| \leq G(x), \quad \text { for all } n \in \mathbb{N} \quad \text { and } \quad x \in \mathbb{R}^{2} .
$$


Since

$$
\left\{\begin{array}{l}
-\Delta v_{n}+V(x) v_{n}=A\left(\epsilon_{n} x+\epsilon_{n} y_{n}\right) f\left(v_{n}\right), \quad \text { in } \quad \mathbb{R}^{2}, \\
v_{n} \in H^{1}\left(\mathbb{R}^{2}\right)
\end{array}\right.
$$

From [10, Theorems 9.11 and 9.13], there exists $C_{3}>0$ independently of $x \in \mathbb{R}^{2}$ and $n \in \mathbb{N}$ such that $v_{n} \in W^{2,3}\left(B_{2}(x)\right)$ and

$$
\|\left. v_{n}\right|_{W^{2,3}\left(B_{2}(x)\right)} \leq C_{3}|G|_{L^{3}\left(B_{2}(x)\right)}, \quad \text { for all } n \in \mathbb{N} .
$$

On the other hand, from continuous embedding $W^{2,3}\left(B_{2}(x)\right) \hookrightarrow C\left(\overline{B_{1}(x)}\right)$, there is $C_{4}>0$ independently of $x \in \mathbb{R}^{2}$ such that

$$
\|u\|_{C\left(\overline{B_{1}(x)}\right)} \leq C_{4}\|u\|_{W^{2,3}\left(B_{2}(x)\right)}, \quad \text { for all } u \in W^{2,3}\left(B_{2}(x)\right) .
$$

The result follows from (3.39) and (3.40).

\section{Concentration of the solutions:}

The proof of the concentration follows with the same idea explored in the case $N \geq 3$, then we omit its proof.

\section{References}

[1] C.O. Alves and G. F. Germano, Ground state solution for a class of indefinite variational problems with critical growth, arXiv:1704.01385v1[math.AP].

[2] C.O. Alves and G. F. Germano, Existence and concentration of solution of ground state solution for a class of indefinite variational problems, arXiv:1801.06872 1 [math.AP].

[3] C.O. Alves, J. M. B. do Ó and O.H. Miyagaki. On nonlinear perturbations of a periodic elliptic problem in $\mathbb{R}^{2}$ involving critical growth. Nonlinear Analysis 45 (2001) 849-863.

[4] D.M. Cao, Nontrivial solution of semilinear elliptic equation with critical exponent in $\mathbb{R}^{2}$, Comm. Partial Differential Equations 17 (1992), 407-435.

[5] J. Chabrowski and A. Szulkin, On a semilinear Schrödinger equation with critical Sobolev exponent. Proc. Amer.Math. Soc. 130 (2001) 85-93. 
[6] D. Cassani and C. Tarsi, A Moser-type inequality in Lorentz-Sobolev spaces for unbounded domains in $\mathbb{R}^{N}$, Asymptotic Anal. 64, 29-51 (2009).

[7] D.G. de Figueiredo, O.H. Miyagaki and B. Ruf, Elliptic equations in $\mathbb{R}^{2}$ with nonlinearities in the critical growth range, Calc. Var. Partial Differential Equations 3, 139-153 (1995).

[8] J. M. B. do Ó and B. Ruf, On a Schrödinger equation with periodic potential and critical growth in $\mathbb{R}^{2}$. Nonlinear Differential Equations Appl. 13 (2006), 167-192.

[9] M.F. Furtado and R. Marchi, Existence of solutions to asymptotically periodic Schrödinger equations, Electronic Journal of Differential Equations, Vol. 2017 (2017), No. 15, 1-7.

[10] D. Gilbarg and N.S. Trundiger, Elliptic partial differential equation of second order. $2^{\text {nd }}$ edition. Grundlehren 224, Springer, BerlinHeidelberg-New York-Tokyo (1983).

[11] S. Ibrahim, N. Masmoudi and K. Nakanishi, Trudinger-Moser inequality on the whole plane with the exact growth condition, J. Eur. Math. Soc. $\mathbf{1 7}(4)(2015), 819-835$.

[12] M. Ishiwata, Existence and nonexistence of maximizers for variational problems associated with Trudinger-Moser type inequalities in $\mathbb{R}^{N}$, Mathematische Annalen 351(2011), 781-804 .

[13] W. Kryszewski and A. Szulkin, Generalized linking theorem with an application to a semilinear Schrödinger equation. Adv. Differential Equations 3 (1998), 441-472.

[14] P. L. Lions, The concentration-compactness principle in the calculus o variations. The limit case, Rev. Mat. Iberoamericana 1 (1985), 145-201.

[15] G.B. Li and A. Szulkin, An asymptotically periodic Schrödinger equation with indefinite linear part. Commun. Contemp. Math, 2002, 4: $763-776$

[16] A. Pankov, Periodic nonlinear Schrödinger equation with application to photonic crystals, Milan J. Math. 73 (2005) 259-287.

[17] A. Pankov and K. Pflüger, On a semilinear Schrödinger equation with periodic potential, Nonl. Anal. TMA 33 (1998), 593-609. 
[18] N. Masmoudi and F. Sani, Trudinger-Moser Inequalities with the Exact Growth Condition in $\mathbb{R}^{N}$ and Applications, J. Comm. in Partial Diff. Eqns 40(2015), 1404-1440 .

[19] M. Ramos, Z-Q. Wang and M. Willem, Positive solutions for elliptic equations with critical growth in unbounded domains, Calculus of Variations and Differential Equations, Chapman \& Hall/CRC Press, Boca Raton, 2000, pp. 192-199.

[20] P.H. Rabinowitz, Minimax methods in critical point theory with applications to differential equations. AMS Reg. Conf. Ser. Math. 65 (1986)

[21] B. Ruf, A sharp Trudinger-Moser type inequality for unbounded domains in $\mathbb{R}^{2}$, Journal of Functional Analysis 219 (2005) 340-367.

[22] A. Szulkin and T. Weth, Ground state solutions for some indefinite variational problems, J. Funct. Anal. 257 (2009) 3802-3822.

[23] X.H. Tang, Non-Nehari manifold method for asymptotically periodic Schrödinger equations, Science China Mathematics 58 (2015), 715-728.

[24] X.H. Tang, New super-quadratic conditions for asymptotically periodic Schrödinger equation, Preprint

[25] H. Zhang, J. Xu and F. Zhang, Ground state solutions asymptotically periodic Schrödinger equations with indefinite linear part, Mathematical Methods in the Applied Sciences 38 (2015), 113-122.

[26] H. Zhang, J. Xu and F. Zhang, On a class of semilinear Schrödinger equation with indefinite linear part, J. Math. Anal. Appl. 414 (2014), 710-724. 